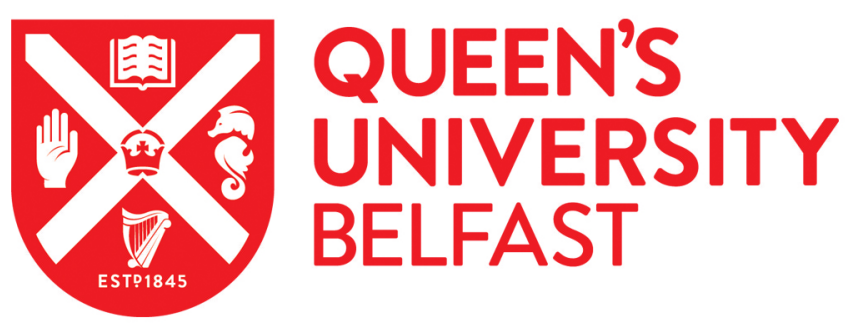

\title{
110th Anniversary: Comparison of Cavitation Devices Based on Linear and Swirling Flows: Hydrodynamic Characteristics
}

Simpson, A., \& Ranade, V. V. (2019). 110th Anniversary: Comparison of Cavitation Devices Based on Linear and Swirling Flows: Hydrodynamic Characteristics. Industrial and Engineering Chemistry Research, 58(31), 14488-14509. https://doi.org/10.1021/acs.iecr.9b02757

Published in:

Industrial and Engineering Chemistry Research

Document Version:

Peer reviewed version

Queen's University Belfast - Research Portal:

Link to publication record in Queen's University Belfast Research Portal

Publisher rights

(c) 2019 American Chemical Society. This work is made available online in accordance with the publisher's policies. Please refer to any applicable terms of use of the publisher.

\section{General rights}

Copyright for the publications made accessible via the Queen's University Belfast Research Portal is retained by the author(s) and / or other copyright owners and it is a condition of accessing these publications that users recognise and abide by the legal requirements associated with these rights.

Take down policy

The Research Portal is Queen's institutional repository that provides access to Queen's research output. Every effort has been made to ensure that content in the Research Portal does not infringe any person's rights, or applicable UK laws. If you discover content in the Research Portal that you believe breaches copyright or violates any law, please contact openaccess@qub.ac.uk. 


\title{
$110^{\text {th }}$ Anniversary: Comparison of Cavitation Devices based on Linear and Swirling flows: Hydrodynamic Characteristics
}

\author{
Alister Simpson and Vivek V. Ranade* \\ School of Chemistry and Chemical Engineering \\ Queen's University Belfast, Belfast BT9 5AG, UK \\ *Email: V.Ranade@qub.ac.uk
}

\begin{abstract}
The interest in cavitating flow reactors has intensified over recent years, and a significant amount of research effort has been focussed on demonstrating the potential applications of hydrodynamic cavitation. The knowledge base on the design of devices to optimise cavitation yield, performance and industrial scalability remains lacking however. It is essential to develop a sound understanding of the key hydrodynamic characteristics of cavitation devices to address these knowledge gaps. This work presents a comprehensive experimental and numerical investigation into the hydrodynamic behaviour of cavitating devices which feature linear and swirling flows. Two of the most commonly utilized cavitation device geometries are studied, namely orifice and Venturi, with and without swirl. These devices are compared to a high swirl flow device (vortex diode). A series of experimental configurations were designed with the aid of multiphase, unsteady computational fluid dynamics simulations, so as to achieve matching power input in terms of flow rate versus pressure drop across all 5 device configurations, allowing their cavitation characteristics to be directly compared on a consistent basis. High speed flow visualisation and detailed numerical predictions are presented which clearly describe the influence that key parameters such as swirl ratio and Reynolds number have on the nature of the observed cavitating flow structures. Cavitation inception conditions are described for each device, with Venturi and vortex devices shown to generate incipient cavitation at lower pressure ratios than orifice devices. Cavitation numbers are computed, which indicate that values of unity are obtained at inception across the range of devices provided that appropriate characteristic velocities are defined. Swirl is identified as an important parameter in cavitation device design, with the swirling flow device designs shown to successfully move the cavitating region away from solid surfaces towards the device axis. Importantly, this is achieved without an energy consumption penalty; the results describe how swirl can be utilized to design devices which minimise or eliminate the risk of surface erosion.
\end{abstract}

Key words: Hydrodynamic cavitation, orifice, Venturi, vortex diode, CFD, multiphase 
Cavitation is a process of generation, growth and subsequent collapse of gas filled cavities. The extreme physio-chemical effects produced by cavitation have been of interest to engineers and scientists for over a century, and the concept of cavitation damage predates the 110 year history of the journal of Industrial Engineering Chemistry Research. In 1885 the phenomenon of cavitation was proposed as the reason behind the sea trial failure of the propellers on the British high speed warship, the HMS Darling. The mechanisms of cavitation damage have been debated for a century or since, and to this day the causes and effects still present fundamental scientific challenges for engineers; continuing issues in the field of water turbines are best exemplified by the "three gorges puzzle" ${ }^{1}$. The explosive mechanisms of cavitation were first suggested by Lord Rayleigh in $1917^{2}$, which forms a basis for over 100 years of study into the subject. Rayleigh considered the scenario of the collapse of an "empty" spherical cavity in an incompressible fluid, assuming that the pressure at a distance from the bubble remained constant. With this assumption, Rayleigh was able to calculate the motion of the bubble free surface as a function of time.

This basic approach has been iteratively improved over the years to relax many of the simplifying assumptions and take into account additional physical mechanisms ${ }^{3-6}$, however Rayleigh's initial hypothesis was fundamental in helping to shape the early understanding of the physical effects of cavitation. The non-linear growth of cavitation bubbles posited by Rayleigh was demonstrated in pioneering work published by Knapp and Hollander in $1948^{7}$. The authors studied the formation \& collapse of cavitation bubbles in an unprecedented level of detail, using a high-speed photography system capable of capturing 20,000 frames/s. Comparison of these measured results with the Rayleigh equation over the initial growth and collapse period showed remarkable agreement, both in bubble radius and radial velocities. The initial bubble growth phase was found to be relatively steady, whereas the collapse and subsequent rebound stages indicate extremely rapid acceleration. These rapid accelerations during the collapse phases are understood to produce highly localised spots of extreme temperatures and pressures $\left(5000^{\circ} \mathrm{K}\right.$ and $1000 \mathrm{~atm}$ respectively $\left.{ }^{8,9}\right)$. These collapse events are accompanied by localised shock waves and extremely high shear rates, and as a result cavitation can be famously damaging in pressurised fluid handling equipment, with a large number of historical studies devoted to material damage mechanisms (see for example early work presented by Plesset and Ellis in $1955^{10}$, Eisenberg in $1963^{11}$ and Hammit in $1965^{12}$, as well as recent reviews by Dular et al. ${ }^{13}$ and Sreedhar et al. ${ }^{14}$ ). The extreme conditions generated during cavitation can however also be harnessed for realising beneficial physio-chemical transformations. Collapsing cavities generate extreme pressure, temperature, intense shear and oxidising radical species, including hydroxyl radicals $(\mathrm{OH})$, which can be utilized for a variety of applications including advanced oxidation processes.

The successful application of hydrodynamic cavitation $(\mathrm{HC})$ has been widely reported for a broad range of industrially relevant processes, such as microbial disinfection ${ }^{15}$, effluent treatment ${ }^{16}$, wastewater sludge treatment ${ }^{17}$, bio-mass pre-treatment ${ }^{18,19}$, fuel desulfurization ${ }^{20}$, biodiesel synthesis ${ }^{21,22}$, and in food and beverage production ${ }^{23,24}$. Further comprehensive summaries can be found in the reviews compiled by Rajoriya et al. ${ }^{25}$, Dular et al. ${ }^{26}$ and Ranade and Bhandari ${ }^{27}$. The Interest in cavitating flow reactors has intensified over recent years, and cavitation has been identified as a very promising technology to enhance a wide range of physio-chemical processes ${ }^{28}$. Despite the intense interest and significant number of publications on cavitation, adequate information on the design of cavitation reactors is not available. While a significant amount of research effort has been focussed on demonstrating the potential application of hydrodynamic cavitation, the knowledge base on the design of devices to optimise cavitation yield, performance and industrial scalability remains lacking. In order to realise the true potential of hydrodynamic cavitation, it is essential to develop a sound understanding of the key hydrodynamic characteristics of cavitation devices. 
Cavitation devices may be broadly grouped into two types: devices based on moving parts and devices without any moving parts. The first class of devices are typically comprised of a rotor - stator assembly. These are rather expensive to construct, incur a higher maintenance overhead and therefore are suitable only for a few high value applications. Cavitation devices without moving parts have significantly lower costs, and are considered to be the most promising route to realising the full potential of hydrodynamic cavitation, particularly for large scale applications. Cavitation devices without moving parts can be further classified into two broad types (and their possible combinations): those based on linear flow, and those based on swirling flows. These devices and their corresponding low pressure, cavitation zones are shown schematically in Figure 1.

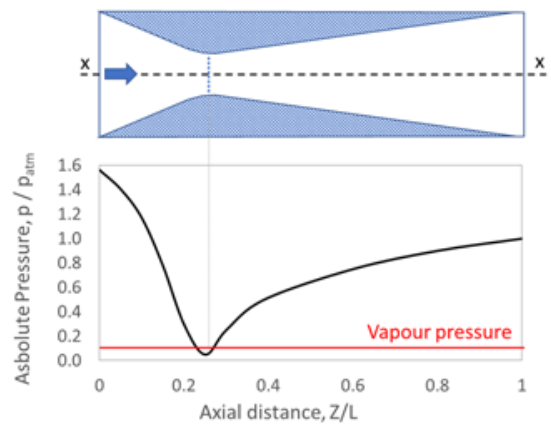

(a) Linear flow
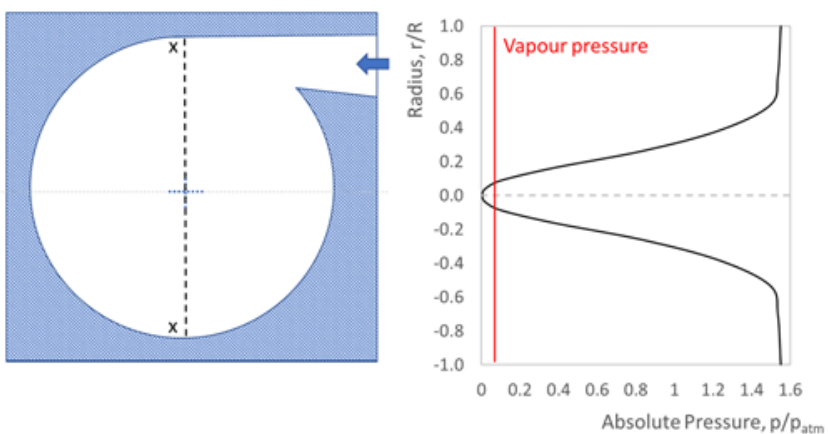

(b) Swirling flow

Figure 1: Schematic of pressure distributions in linear flow (a) and swirling flow (b) devices

In linear flow cavitation devices such as an orifice or Venturi, the working fluid is forced through a restriction, such that the local acceleration and associated static pressure reductions generate pressures close to or below the saturated vapour pressure (Figure 1a). In a swirling flow device, provided that there is a sufficiently high swirl component, a low-pressure cavitating region is generated at the swirl axis (Figure 1b). In this second scenario, the cavitating zone can be sufficiently removed from solid surfaces so as to eliminate the risk of associated damage to the device, and swirl is therefore a potentially useful parameter in designing devices which can be used reliably within an industrial process environment.

The available literature features the use of a wide range of variants of these main types of device to carry out a range of physio- chemical transformations. To highlight some specific examples; Capocelli et al. ${ }^{29}$ have reported the use of a linear Venturi reactor to successfully degrade $p$-nitrophenol. Using a $2 \mathrm{~mm}$ throat diameter device, the authors achieved maximum removal percentages of the order of $40 \%$. The influence of pressure ratio was found to be significant; optimum degradation was found at a pressure drop of $450 \mathrm{kPa}$, with performance then found to drop off significantly, with just $10 \%$ degradation found at a marginally higher pressure drop of $500 \mathrm{kPa}$. Also studying p-nitrophenol degradation, Pradhan \& Gogate ${ }^{30}$ compared results using both linear flow orifice and Venturi devices. Maximum removal rates were observed for the Venturi of the order of $45 \%$, with the orifice yielding marginally lower degradation by the order of $3 \%$ at the same operating pressure. Other researchers have studied the influence of orifice geometry on performance; for example Sivakumar \& Pandit ${ }^{31}$ have reported the degradation of Rhodamine B dye using a variety of orifice plate configurations featuring multi-hole designs, concluding that a larger number of smaller holes can lead to improved results. Promising performance results have also been published for novel, swirling flow type reactor designs, such as the Ecowirl ${ }^{\circledR}$ reactor described by Mancuso ${ }^{32}$. Also studying Rhodamine $B$ as the pollutant, Mancuso et al. ${ }^{33}$ have presented improved results using an Ecowirl ${ }^{\circledR}$ reactor in comparison to orifice type configurations. Wang et al. ${ }^{34}$ have achieved yet further improvements in Rhodamine B removal using a swirling flow cavitation device. Novel, vortex diode type reactors have also recently yielded promising results in the degradation of industrial effluents; Suryawanshi et al. ${ }^{35}$, and 
Sarvonathan et al. ${ }^{36}$ have demonstrated the effectiveness of a vortex diode in removing solvents from wastewater. Swirling flow reactors have also found use in bacterial disinfection applications; the use of a high swirl, vortex diode device has recently been presented by Jain et al. ${ }^{37}$, which was found to efficiently eliminate both $\mathrm{E}$-coli and $\mathrm{S}$. aureus. The authors also compared their results to a linear flow orifice device, and found that the orifice required significantly higher energy input in order to obtain the same level of reactor performance. Loraine et al. $^{38}$ have presented exciting results on the reduction of both E-coli and B-subtilis, achieving reductions of $\log 7$ and $\log 4.5$ respectively using a DynaJet $^{\circledR}$ system. On comparison with ultrasonic methods they found an improvement in energy efficiency in excess of a factor of 10. Also. Another promising application for cavitating swirl flow devices is in fuel desulfurization, in which recent studies have yielded successful conversion results ${ }^{20,39}$.

Despite several published studies on these cavitation devices, any attempt at a direct comparison of the results achieved with different devices is almost impossible due to a lack of any consistent basis for such comparison. The cavitation state within a device is often described by the cavitation number, $\sigma:$

$$
\sigma=\frac{p_{d}-p_{v}}{1 / 2 \rho_{l}(u)^{2}}
$$

Where $p_{d}$ and $p_{v}$ are the discharge and vapour pressures respectively, $\rho_{l}$ is the liquid density, and $u$ is a characteristic velocity, typically defined as the device throat velocity. In general, the lower the cavitation number, the higher the cavitation intensity. There are however well documented inconsistencies with how this parameter is reported across the available literature, particularly around the specification of a suitable characteristic velocity. The limitations in using cavitation number to compare or define performance have been summarised in detail by Sarc et al. ${ }^{40}$. Even for the same type of device, small changes in configuration can have a significant impact on the cavitation behaviour; for example relatively small variations in orifice hole geometry can significantly affect cavitation inception characteristics ${ }^{41}$. Similarly, changes to the geometry of Venturi devices also have a significant influence on cavitation behaviour ${ }^{42}$. The information on devices which combine rotational and linear flows is almost non-existent, although in a recent study on a cavitating Venturi Kozak et al. ${ }^{43}$ have highlighted the significant influence that the introduction of swirl can have on the cavitating flow structures. The primary objective of this work is for the first time, to compare cavitation devices based on linear flows (orifice and Venturi) and swirling flows (vortex diode) on a systematic basis. The use of swirler device placed in-line before the orifice or Venturi was also investigated in order to clearly understand the differences and similarities of the hydrodynamics of cavitation devices based on linear and swirling flows. The key role of swirling flows on influencing the characteristics of cavitation is highlighted.

A uniform basis is developed to design different cavitation devices based on linear flows, rotational flows and their combination. These cavitation devices were then fabricated using acrylic in order to facilitate detailed high-speed flow visualisation. Systematic experimental investigations were carried out to compare the key characteristics of hydrodynamic cavitation realised in these different devices. Cavitation inception for different devices was identified and measured. High speed visualisation was used to capture key characteristics of cavitation. Comprehensive computational fluid dynamic models have been developed and applied to simulate hydrodynamics of the considered devices at a wide range of operating conditions and swirl ratios. Simulated results using the developed computational models were compared with the experimental data. The validated models were then used to understand the influence of key design parameters, such as Reynolds number and swirl ratio, on the generated hydrodynamic cavitation. An attempt is made for the first time to systematically discuss the differences and similarities of cavitation realised by linear and swirling flows. The developed approach, models and presented results will be useful for designing improved hydrodynamic cavitation devices by appropriate combinations of linear and swirling flows. 


\section{Materials and Methods}

\subsection{Hydrodynamic Cavitation Devices}

It is important to establish a common basis for comparing hydrodynamics of cavitation devices based on linear and swirling flows. We selected the vortex diode as a characteristic cavitation device based on swirling flows (Ranade et al. ${ }^{44}$ ). The device geometry is shown in Figure $2 \mathrm{a}$, with the throat diameter selected as $12 \mathrm{~mm}$. The two most widely used hydrodynamic cavitation devices based on linear flows, namely orifice and Venturi, were selected for comparison with the vortex device. As discussed in the previous section, while data is available in open literature comparing the reactor performance of different device geometries at similar pressure drops, the flow rates, velocities and cavitation numbers vary appreciably between configurations. Considering that the cavitation devices are directly compared on the basis of cost for a given throughput, devices to be compared should have similar power consumption (product of flow rate and pressure drop, $Q . \Delta p$ ) across the operating range. This in turn allows hydrodynamic characteristics, such as the turbulent flow properties, and ultimately the device performance, to be compared on a consistent basis. As such the initial experimental design challenge was to determine dimensions of orifice and Venturi which provided matching pressure drop versus flow rate characteristics to the existing vortex diode. Following previous investigations into cavitating orifice and Venturi devices ${ }^{37,36}$, the basic geometrical parameters such as the Venturi nozzle and diffuser wall angles $(\alpha$ and $\beta)$, and the orifice throat diameter to length ratio $\left(I / d_{t}\right)$ were selected. From that starting point, a series of CFD calculations were then performed while adjusting the throat diameter, $d_{t}$, for each device until the predicted flow rate versus pressure drop was found to overlap that of the measured and predicted data for the vortex diode. This required three iterations per device, and full details of the computational approaches used throughout the present study are briefly in the subsequent sections. The resulting flow characteristic plots for each device are shown in Figure $2 \mathrm{~b}$, and the resulting final dimensions are tabulated in Figure $2 \mathrm{a}$. 

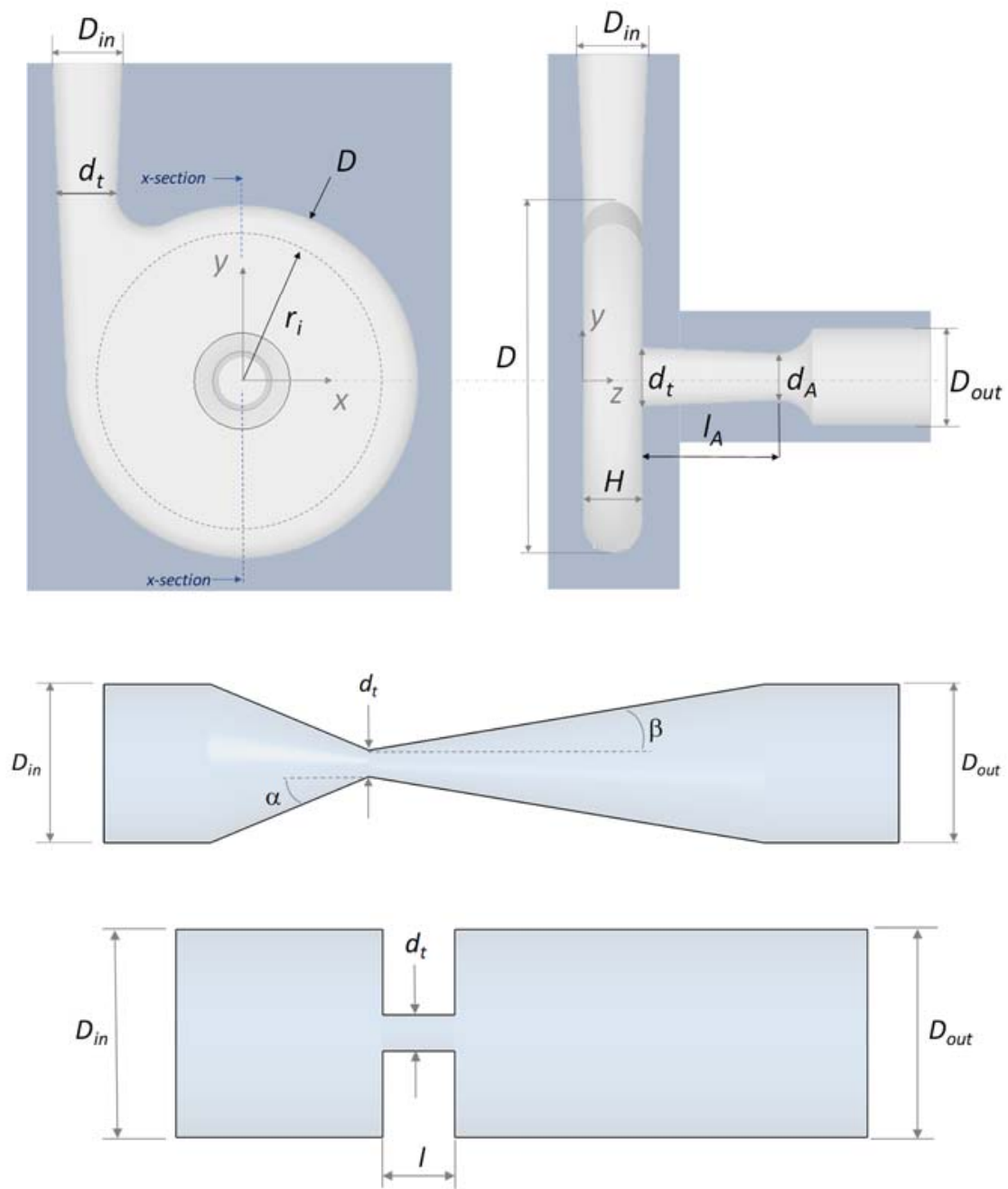

(a) Device geometries (not to scale)

\begin{tabular}{|l|c|}
\hline$D[\mathrm{~mm}]$ & 72.0 \\
\hline$D_{\text {in }}[\mathrm{mm}]$ & 18.2 \\
\hline$r_{i}[\mathrm{~mm}]$ & 30.0 \\
\hline$d_{t}[\mathrm{~mm}]$ & 12.0 \\
\hline$d_{A}[\mathrm{~mm}]$ & 9.6 \\
\hline$D_{\text {out }}[\mathrm{mm}]$ & 18.2 \\
\hline$I_{A}[\mathrm{~mm}]$ & 27.2 \\
\hline$H[\mathrm{~mm}]$ & 12.0 \\
\hline
\end{tabular}

\begin{tabular}{|l|c|}
\hline$D_{\text {in }}[\mathrm{mm}]$ & 25.4 \\
\hline$\alpha^{\circ}$ & 22.5 \\
\hline$d_{t}[\mathrm{~mm}]$ & 4.0 \\
\hline$\beta^{\circ}$ & 9.25 \\
\hline$D_{\text {out }}[\mathrm{mm}]$ & 25.4 \\
\hline
\end{tabular}

\begin{tabular}{c|c|}
\hline$D_{\text {in }}[\mathrm{mm}]$ & 25.4 \\
\hline$d_{t}[\mathrm{~mm}]$ & 5.0 \\
\hline$I[\mathrm{~mm}]$ & 10.0 \\
\hline$D_{\text {out }}[\mathrm{mm}]$ & 25.4 \\
\hline
\end{tabular}

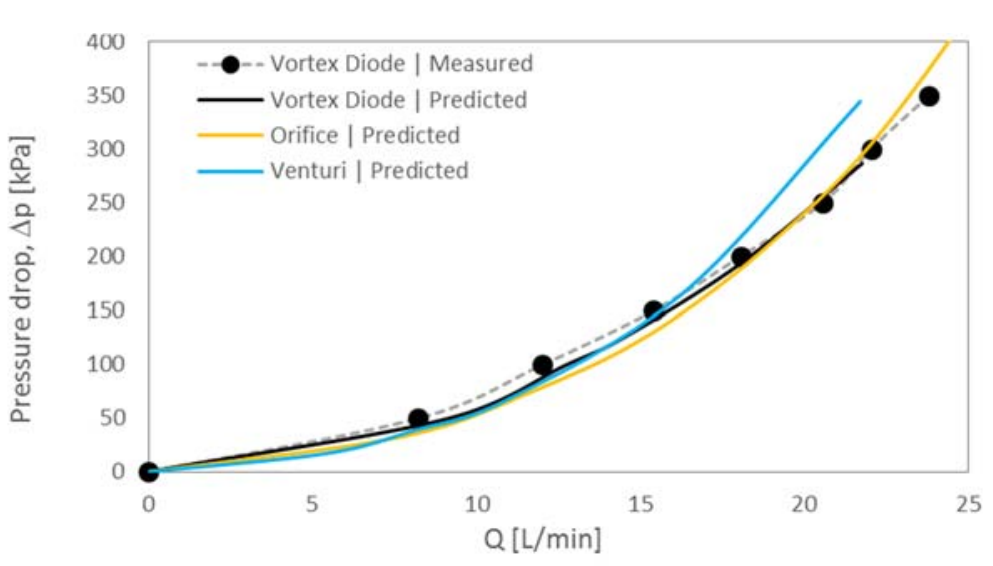

(b) Pressure drop and flow rate relationships for vortex diode, Venturi and orifice

Figure 2: Cavitation device geometries \& operating conditions

The vortex diode is characterised by high swirl ratios (Pandare and Ranade, 2015 ${ }^{46}$; Simpson and Ranade, $2019^{47}$ ), with the maximum tangential velocities locally reaching values in excess of five times the linear velocity at the throat of the axial port. In order to understand and compare cavitation devices based on swirling and linear flows, an in-line swirl device (swirler) was designed and installed upstream of orifice and Venturi. The swirler was designed so as to experimentally study intermediate swirl ratio values, and also to directly compare cavitating flows with and without swirl. The basic 
design comprised a series of guide vanes which turn the flow from the purely axial direction to the tangential direction. The detailed design of the guide vane profiles was carried out using Ansys BladeGen software. The blade angles and thicknesses for each section are defined by Bezier curves as shown in Figure 3a, with the final swirler comprising of 5 vanes in total arranged around a profiled hub body (Figure $3 b$ ). The final blade profiles were again determined iteratively to maximise the tangential velocities without excessive additional pressure losses. The final design features five guide vanes with outlet angle $50^{\circ}$ at the hub $(10 \mathrm{~mm} \phi)$, up to $80^{\circ}$ at the blade tip. The guide vane body was made with nylon by 3D printing (Figure 3c). The key dimensionless numbers (Reynolds number, Re and Swirl number, S) are defined as:

$$
R e=\frac{\rho \overline{u_{t}} d_{t}}{\mu}
$$

$$
S=\frac{u_{\theta \max }}{\overline{u_{t}}}
$$

The characteristic velocity scales and length scales used for defining Re and S are shown in Figure $3 d$ and swirl ratios are shown in Figure $3 \mathrm{e}$. Re is based on mass averaged velocity at throat and throat diameter, $d_{t}$, for each device. The $S$ is based on maximum tangential velocity and the mass averaged velocity at throat. The swirler used in this work delivered a swirl ratio, $\left(u_{d} / u_{z}\right)$ in excess of 2 at the outlet of swirler. The swirl ratio reduces to values of the order of 0.5 at the throat as flow is accelerated through the restriction (see Figure 3e). The fixed swirler geometry limits the experimental exploration to a single value of swirl ratio for each device; in order to widen the study to explore intermediate values of swirl ratio beyond that covered by the experimental configurations, the numerical investigation was extended in order to elucidate the changes in flow behaviour across a wider range of inlet swirl ratio to the Venturi and orifice (see Section 5.6 for full details).

\subsection{Cavitation test rig \& procedure}

The experimental facility is shown schematically in Figure 4, consisting of a centrifugal pump (Pedrollo $3 \mathrm{C}$ R80), which is gravity fed from a $10 \mathrm{~L}$ holding tank. The tank incorporates a heat exchanger which was set to maintain temperatures of $22^{\circ} \mathrm{C}$ throughout the present series of tests. The pipework consists of 1" U-PVC section, with a bypass line and system of manual ball valves used to set the flow rates and pressures through the cavitating devices. The cavitation devices are each interchangeable, and installed in the main line using union couplings. Flow rates measurement was carried out using a calibrated variable area flow meter installed in line with the device, and inlet static pressure was measured using a static pressure gauge installed in a t-section upstream of the device inlet as shown in Figure 4a. Gauge (relative) pressures were recorded upstream and downstream of the device; throughout the tests the outlet valve was fully open, and as a result the device discharged to atmospheric pressure for each device configuration. The Venturi, orifice and vortex devices were all machined from acrylic in order to facilitate detailed flow visualization using a high-speed camera system. The Venturi was machined as a single component, whereas the orifice consisted of a cylindrical housing which can facilitate different orifice plate configurations. Due to its relative complexity the vortex unit was machined as four separate components; a two-part split chamber housing, with separate inlet and axial ports.

Flow visualization was performed using a high-speed digital camera (OPTRONIS CP90-3-M-540), fitted with a precision lens producing a resolution of $1200 \times 1200$ pixels at relatively short minimum working distances $(20 \mathrm{~mm})$. Image capture carried out using a frame grabber (SISO Microenable 5 AQ8-CXP6D) installed in a dedicated image data acquisition PC, with the system capable of recording full resolution images at the maximum camera frame rate of $550 \mathrm{fps}$. Lighting was provided by a $25 \mathrm{~W}$ blue LED light source $(465 \mathrm{~nm})$, with the typical lighting arrangement shown in Figure 4a. For each device configuration, image data acquisition was carried out with the light source positioned behind the device relative to the camera. Figure $4 b$ then illustrates the viewing regions for each device 
configuration. As backlighting is used, this results in images in which the secondary vapour phase appears as darker regions. For the vortex diode additional recordings were made at the rear of the diode chamber so as to capture cavitation inception; in this case the light source was positioned behind the camera, resulting in the cavitating core appearing as lighter regions against a dark background. The resulting images presented throughout the manuscript are corrected for light intensity, so that the measured grey intensity level is equalized under non-cavitating conditions (no secondary phase present) for each device. No other corrections have been applied to the images presented.

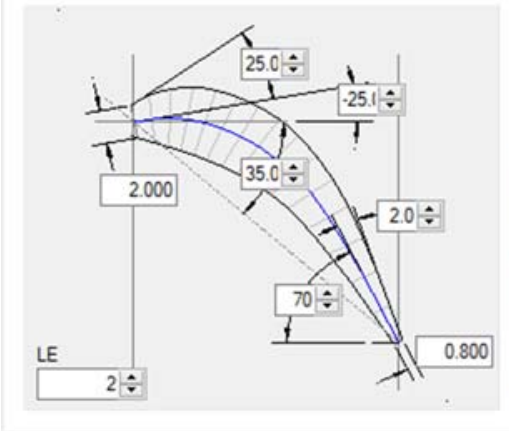

(a) Vane profile design

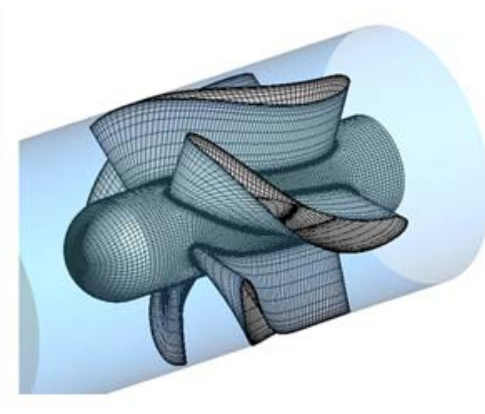

(b) Full 3D guide vane

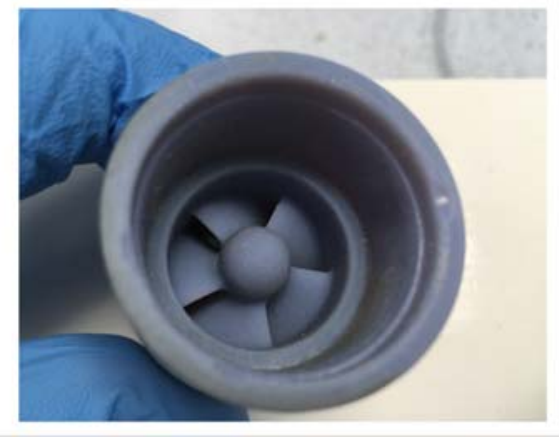

(c) 3D printed component

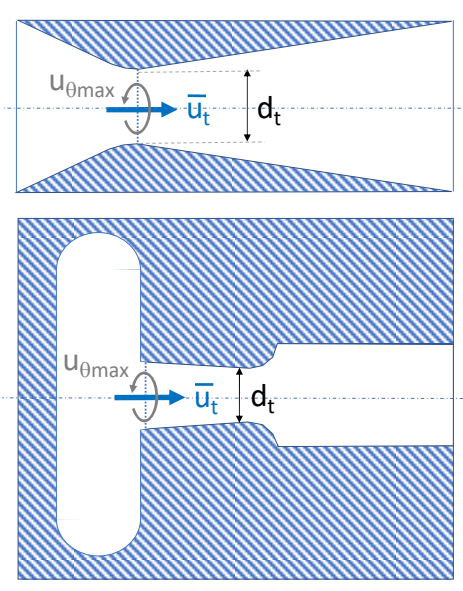

(d) Characteristic velocities

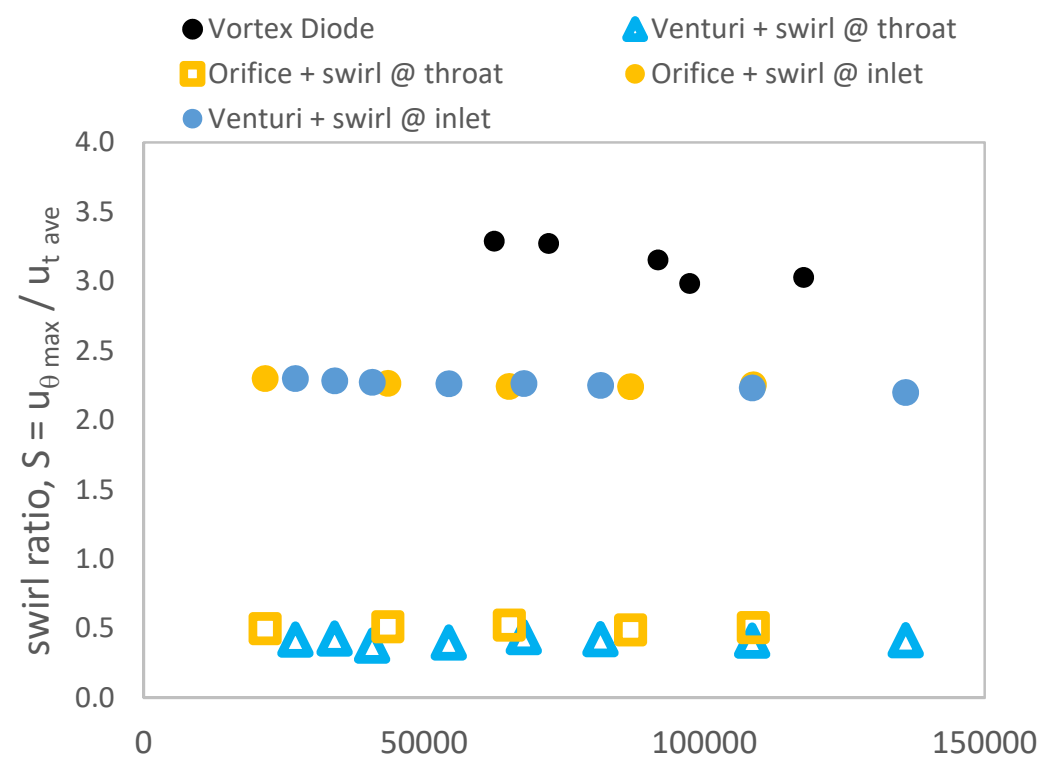

Reynolds number, Re

(e) Device swirl ratios

Figure 3: In-line swirler design

The first step in the experimental investigation was to measure the flow rates and pressure drops across each of the 5 device configurations. In each case the inlet pressure to the device was increased from zero up to a maximum of $350 \mathrm{kPa}$ in increments of $25 \mathrm{kPa}$, and the corresponding flow rate recorded. This procedure was repeated three times for each setup, and the data presented in the subsequent sections is taken from the average of the 3 values. Prior to commencing the cavitation experiments the system was filled with water and left to circulate at non-cavitating conditions, with care taken to ensure any trapped air in the system had been expelled. Repeat measurements of dissolved oxygen were taken using a DO probe, and experiments proceeded only once it was verified that the oxygen content had settled at an equilibrium concentration of $8 \mathrm{~g} / \mathrm{m} 3$ (8ppm). Cavitation 
inception in each case was determined principally by visual observation; noise levels were also monitored manually by stethoscope. Flow rates and pressures were slowly increased in small increments of $5 \mathrm{kPa}$ until visual signs of cavitation were observed with the aid of the high-speed camera focussed on the inception region. In most cases the first appearance of cavitation was accompanied by a significant increase in noise level easily observed by ear. Once the inception operating conditions had been established by repeated testing, camera data acquisition was carried out. Starting at the inception point AVI recordings were carried out at maximum frame rates of $550 \mathrm{fps}$, for an average of 4 seconds at each operating condition. Subsequent post-processing of the recorded image and AVI files was performed using the FIJI distribution of the open source software suite, Image ${ }^{48}$.

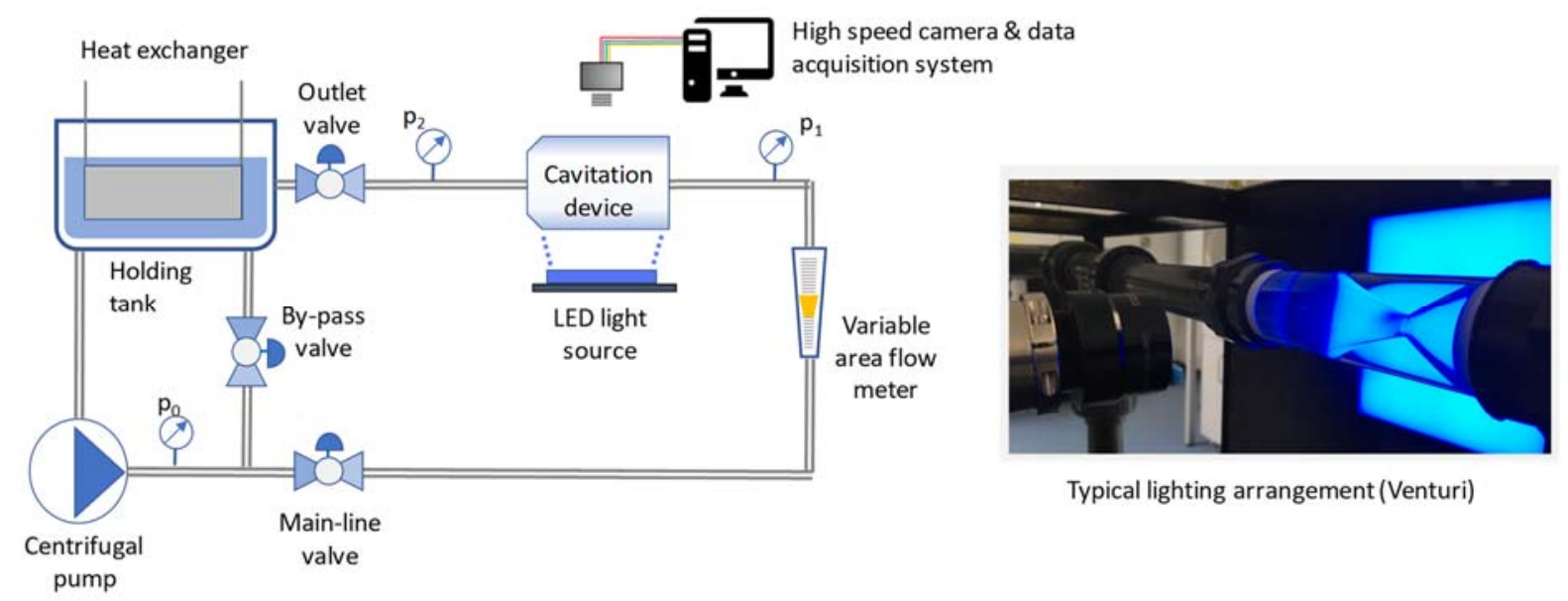

(a) Cavitation test loop schematic
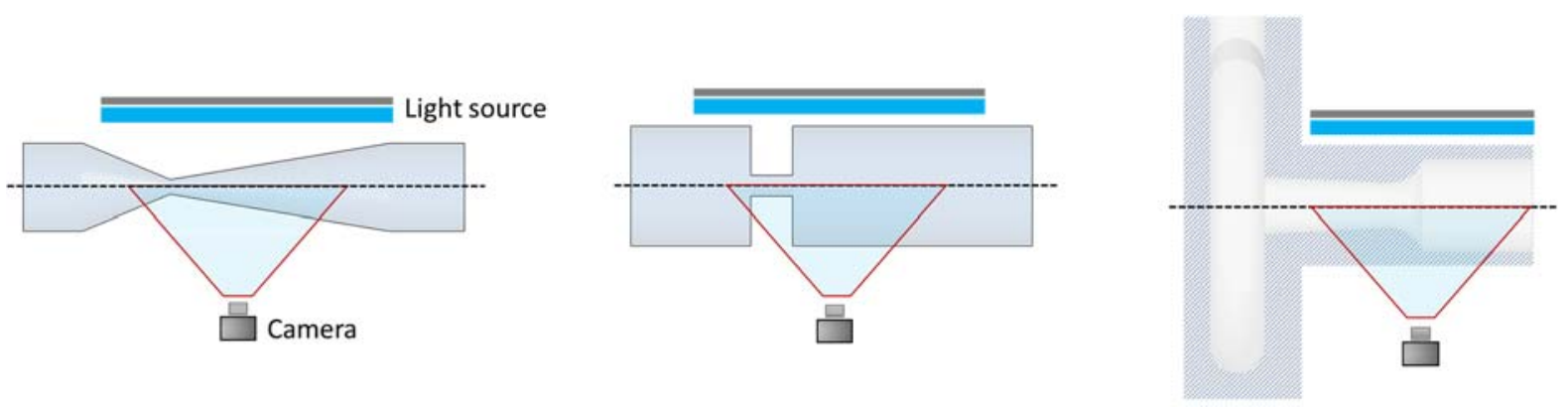

(b) Camera viewing positions

Figure 4: Cavitation test rig

\subsection{Numerical models \& boundary conditions}

The two-phase flow in cavitation devices was modelled by assuming a homogeneous mixture of water liquid and vapour, with the vapour mass fraction, $f$, computed locally using a separate transport equation:

$$
\frac{\partial}{\partial t}\left(\rho_{m} f\right)+\nabla \cdot\left(\rho_{m} \vec{v}_{m} f\right)=\nabla \cdot(\Gamma \nabla f)+R_{e}-R_{c}
$$

Here $\rho_{m}, \rho_{v}$ and $\rho_{l}$ refer to the densities of the mixture, vapor and liquid respectively. This transport equation includes a pair of source and sink terms for the evaporation $\left(R_{e}\right)$ and condensation $\left(R_{c}\right)$ of the vapour phase. There exists in open literature a variety of approximations for this pair of source terms, for example those proposed by Schnerr and Sauer ${ }^{49}$, Zwart et al. ${ }^{50}$ and Singhal et al. ${ }^{51}$. Based 
on previous studies ${ }^{41,42,47}$, the Singhal model was used in the present study, which is given by Equations (5) and (6).

$$
\begin{aligned}
& R_{e}=C_{1} \frac{\sqrt{k}}{\sigma} \rho_{v} \rho_{l}\left[\frac{2}{3}\left(\frac{p_{v}-p}{\rho_{l}}\right)\right]^{1 / 2}\left(1-f_{v}-f_{g}\right) \\
& R_{c}=C_{2} \frac{\sqrt{k}}{\sigma} \rho_{v} \rho_{l}\left[\frac{2}{3}\left(\frac{p-p_{v}}{\rho_{l}}\right)\right]^{1 / 2} f_{v}
\end{aligned}
$$

The evaporation source term (Equation 4) includes a term for the quantity of non-condensable gas present, $f_{g}$. This is an important parameter in cavitation studies. The total gas content can vary according to a wide range of factors, such as prior liquid processing and storage steps, degassing, pressurization, and the length of time the system has been exposed to air. As previously described, in the current study, it was ensured that water was saturated with oxygen prior to the experiments and the saturated concentration was used as $f_{\mathrm{g}}$.

Turbulent, cavitating flows are known to present unique modelling challenges, and the choice of turbulence model requires careful attention. Cavitating Venturi flows in particular are observed to be inherently transient ${ }^{52-54}$, exhibiting unsteady cavitation cloud shedding downstream of the throat. In a previous study comparing experimental and numerical results for Venturi flows, Reboud et al. ${ }^{53}$ found that standard two equation models, in particular the $k-\varepsilon$ model, tend to over-estimate the turbulent viscosity in the cavitating region, leading to unrealistic predictions of a stable cavity zone. As a remedy they recommended applying an arbitrary pre-multiplier to the turbulent viscosity term, $\mu_{t}$, in order to capture unsteady effects. Arbitrary modifiers of this type have yielded success for other researchers in this field, with a number of examples showing successful capture of cavitation cloud shedding with two equation RANS approaches ${ }^{54-56}$. Similarly, high-swirl ratio cavitating vortices present a different set of modelling challenges, and are also known to exhibit periodic unsteady behaviour ${ }^{57,58}$. Arndt et al. have highlighted some of the complexities in cavitating vortices, which feature unsteady behaviour over a broad frequency spectra, accompanied by complex cavity collapse characteristics ${ }^{59,60}$. In a previous numerical study on single phase flow in vortex diodes, Pandare et al. ${ }^{46}$ highlighted the presence of a periodic, precessing vortex, exhibiting frequencies of the order of $60 \mathrm{~Hz}$. These authors carried out a comparison of the k- $\omega$ SST, re-normalised group (RNG) k- $\varepsilon$ as well as a Reynolds Stress Model (RSM). The $k-\varepsilon$ model was found to underpredict the swirl velocity magnitudes, while the k- $\omega$ SST and RSM models showed close agreement, successfully capturing transient oscillations.

Previous numerical studies in our group of cavitating flows in 2D orifice ${ }^{36}$, Venturi $^{42}$ and vortex $x^{47,61}$ geometries have found the $k-\omega$ SST model to yield more accurate results than $k-\varepsilon$ based models, and to capture key experimental trends in cavitating behaviour. The k- $\omega$ SST model is therefore adopted again in the present study, as this has been shown to provide comparable predictions to more computationally expensive Reynolds Stress Models, provided that sufficient care is taken to mitigate against over-prediction of turbulence viscosity. Standard two equation models can produce excessive turbulence damping, particularly in stagnation regions. The orifice and Venturi geometries both feature some level of flow separation downstream of the restriction, and the vortex diode additionally features a stagnation zone in the vortex core; additional measures were therefore applied here to mitigate against overprediction of the turbulence production term, $G_{k}$. The limiting formulations for the production term, $G_{k}$, proposed by Menter ${ }^{62}$, has been applied as given in Equation (7).

$$
G_{k}=\min \left[G_{k}, C_{l i m} \rho k\right]
$$


Where the limiting coefficient, $C_{\text {lim }}$ is set as 0.9 in the original publication. An additional limiting function, proposed by Kato and Laundeer ${ }^{63}$, has also been applied. This is based on the observation that high turbulence kinetic energy production in stagnation regions is generally caused by the calculation of excessive shear strain rate, $\gamma$. As such, this is corrected by the local vorticity rate, $\Omega$, which tends to low values in stagnation regions:

$$
G_{k}=\mu_{t} \gamma \Omega
$$

Boundary conditions for each of the linear flow cases, the cases with swirler, and the vortex diode were all set with normal velocity inlet and an average pressure outlet, positioned at least 10x diameters downstream of the throat. In order to extend the study to look at intermediate values of swirl between the high swirl diode and the geometries with swirl guide vanes, an additional set of simulations were performed on the orifice and Venturi geometries by introducing a swirl inlet boundary condition as shown in Figure 5. For this part of the study, the inlet tangential velocity was set by applying a rotational speed, set by $u_{\theta \max } / r_{\max }$, superimposed on an axial inlet velocity, $u_{z}$. This allowed the ratio of tangential velocity to axial velocity at the inlet plane to be arbitrarily adjusted. Simulations were then performed for a range of inlet swirl ratios $\left(u_{\theta \max } / u_{z}\right)$ up to values of 16 . In total over sixty 3D, transient, multiphase CFD simulations were performed as part of this study. The numerical results and insights provided by these predictions, along with the accompanying experimental data are presented in the following results and discussion section.
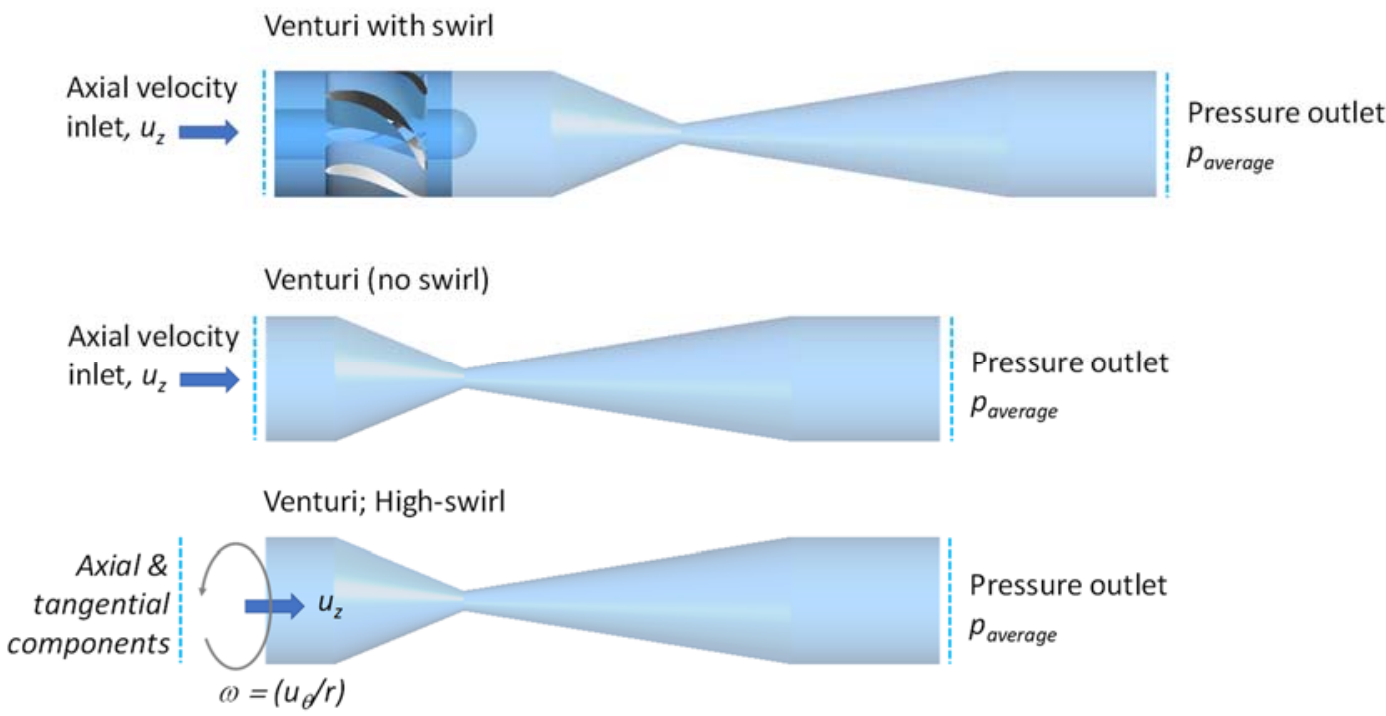

Figure 5: Boundary condition recipes, linear \& swirling flow cases

\subsection{Solution of model equations}

The model equations were solved using a coupled, 3D unsteady solver of the commercial code Ansys Fluent (v17.0). The meshes for each configuration were generated for the most part using Ansys ICEM CFD, with fully hexahedral grids generated. One exception was the swirler, which was meshed separately using Ansys TurboGrid, and subsequently coupled to the downstream orifice and Venturi domains using a general grid interface (GGI). Care was taken in each instance to ensure low $\mathrm{y}^{+}$values, with values in the throat and cavitating regions of each device maintained below 5 . A comprehensive study was carried out to determine the sensitivity of the computed results to number of mesh elements; meshes ranging from 0.5 to $2 \times 10^{6}$ elements were considered for the orifice and Venturi configurations (excluding swirler), and up to $6 \times 10^{6}$ elements were studied for the vortex diode. The vortex diode was found to be the most computationally demanding configuration; detailed surveys 
of the predicted internal velocity gradients and turbulence quantities revealed that mesh sizes of the order of $4 \times 10^{6}$ elements were necessary in order to adequately resolve the key gradients. Further details of the mesh sensitivity study are provided in the supplementary information. Similar studies for the Venturi and orifice configurations resulted in final mesh sizes of $2 \times 10^{6}$ elements, with the final swirler mesh consisting of $0.5 \times 10^{6}$ elements. The final meshed geometries are shown in Figure 6. Besides mesh refinement levels, appropriate consideration was given to final mesh quality; significant meshing effort was focussed on achieving good element quality throughout each domain, with skewness values all below 0.7 .

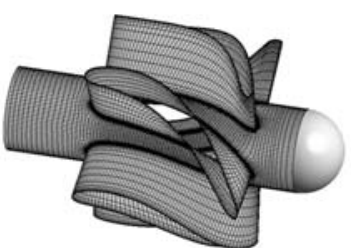

Swirl body: 3D, $4 \times 10^{5}$ hex elements

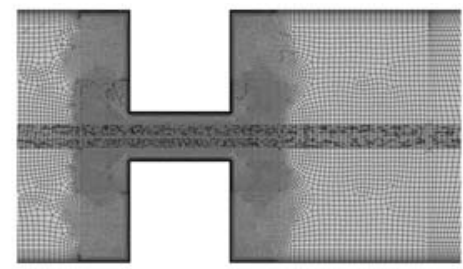

Orifice: $3 D, 2.0 \times 10^{6}$ hex elements

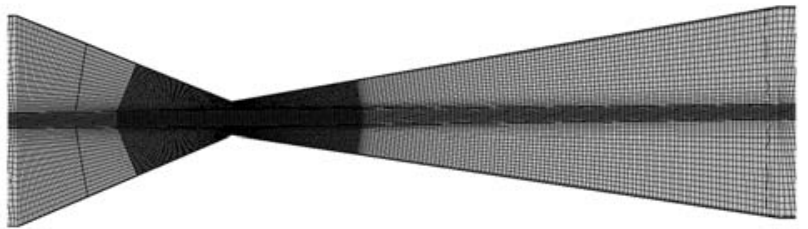

Venturi: 3D, $2.2 \times 10^{6}$ hex elements

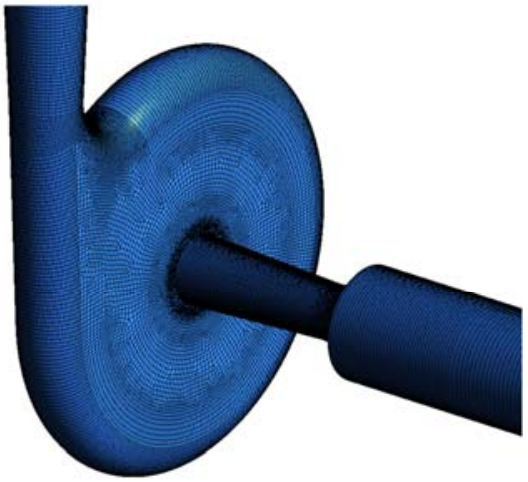

Vortex diode: $3 D, 4.1 \times 10^{6}$ hex elements

Figure 6: Computational grid details

Transient simulations were performed using the coupled solver; pressures were monitored at a number of locations within the throat of each device as well as area averaged values at inlet. Most cases studied here exhibited periodic, transient fluctuations in the monitored flow quantities, such as those presented for the linear flow Venturi in Figure 7; in the cavitating regime predictions showed a periodically advancing and retreating vapour region in the diffuser of the Venturi. Similarly, the vortex diode exhibited unsteady behaviour, with the cavitating vortex core found to oscillate around the swirl axis. The vortex diode was the most computationally intensive, with time stepw as low as $1 \times 10^{-5} \mathrm{~s}$ required to obtain stable convergence to a periodic unsteady flow (total simulated times of the order of $1 \mathrm{~s}$ ). Time step sizes of $1 \times 10^{-4} \mathrm{~s}$ were found to be adequate for the Venturi simulations, and similar timestep sizes of the order of $2 \times 10^{-4}$ were applied for the orifice simulations. Appropriate care was taken to ensure that convergence is achieved for every time step. A more detailed discussion of the observed transient features of each device is presented in the subsequent results section.
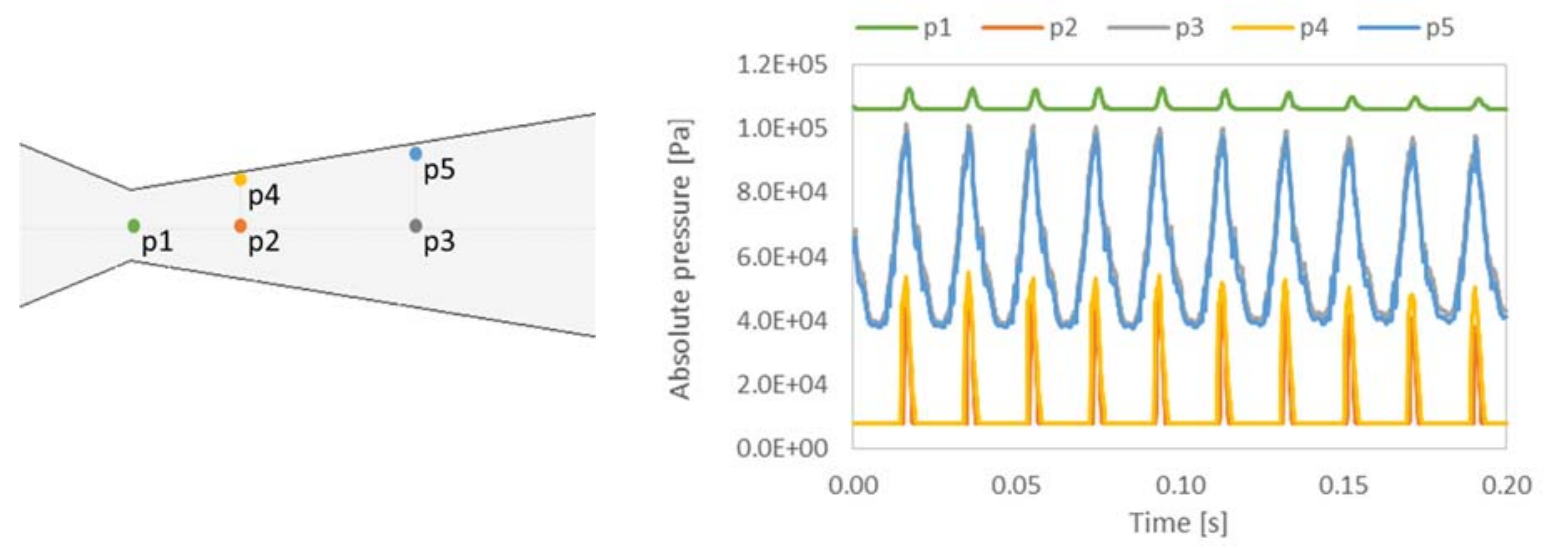

Figure 7: Computed transients, Venturi $(\Delta p=300 \mathrm{kPa})$ 


\subsection{Device Pressure Drop}

The predicted and measured flow rate versus pressure drop data is presented for the vortex diode, Venturi and orifice configurations in Figure 8. The simulated results show reasonably good agreement with the measured data. Beyond a pressure drop of $150 \mathrm{kPa}$, the device characteristics begin to deviate with further increase in flow rate; the Venturi exhibits a steeper increase in pressure losses relative to the vortex unit, whereas the orifice has a relatively flat characteristic curve. These deviations are also captured by the numerical model. For both the orifice and Venturi configurations, the measured flow rate results show a reduction in pressure drop with the introduction of the swirler. This result on its own would suggest that the introduction of this magnitude of pre-swirl acts to suppress turbulence, and reduce dissipative losses downstream of the throat of the device to some extent. Further examination of the computed flow fields do indeed show a slight reduction in turbulence kinetic energy predictions with the experimental swirler arrangement; further details and discussion of the turbulent flow field predictions are presented later in this section. Other researchers have also presented compelling arguments that the imposition of swirl can act to suppress turbulence, and reduce the associated momentum mixing losses ${ }^{64}$. Examining the CFD results for orifice and Venturi in Figure 8 it is evident that the opposite trend is predicted at higher flow rates, where the imposition of swirl results in an increase in losses. This is particularly the case for the Venturi, less so for the orifice predictions. As shown in Figure 7, the venturi exhibits relatively large, transient fluctuations in pressure in the diffuser; the comparison with experimental results would suggest that the resulting calculated turbulence production is excessive at higher flow rates, despite the use of appropriate limiting functions in the closure model.

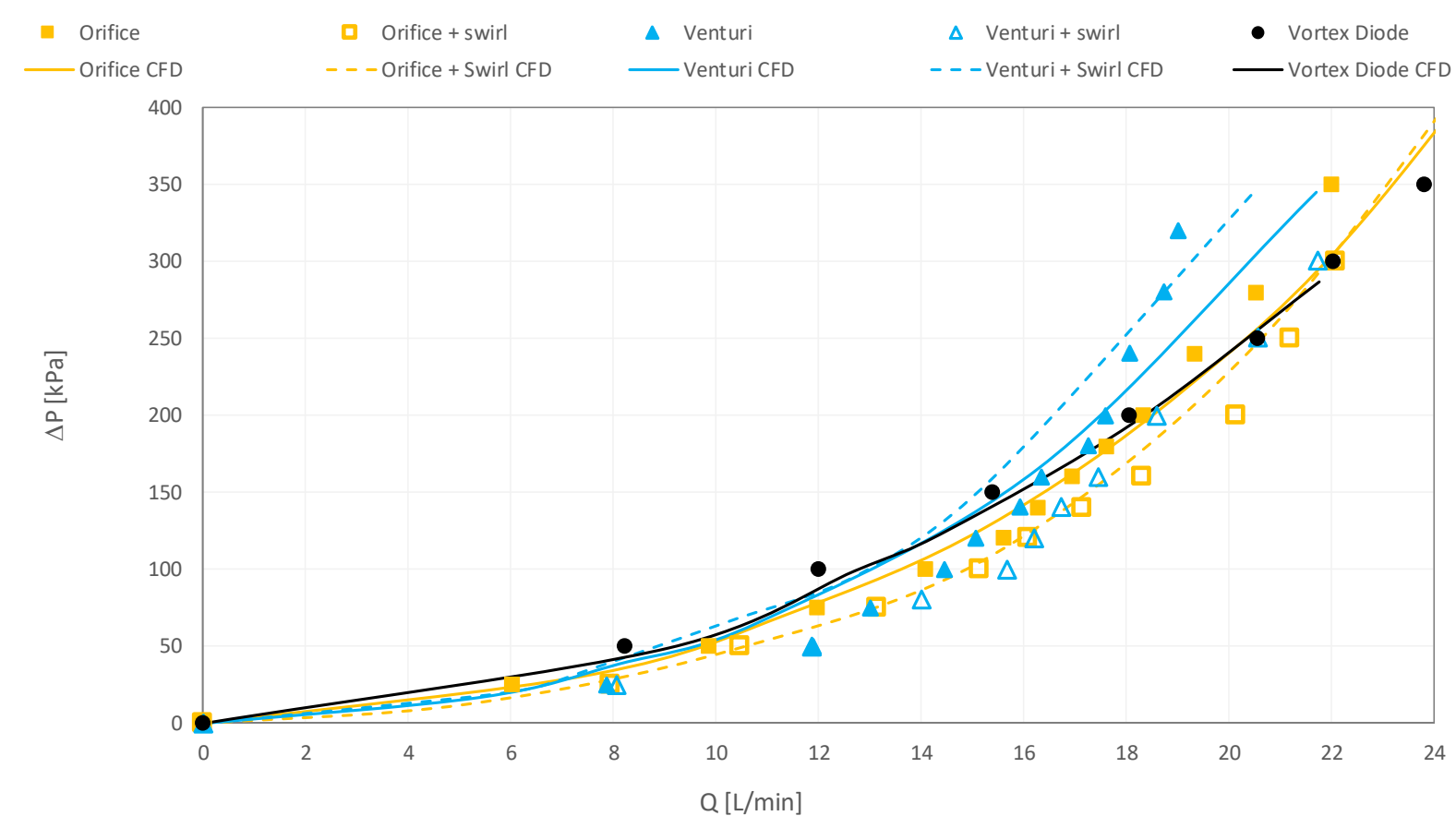

Figure 8: Predicted \& measured flow rate versus pressure drop: Orifice, Venturi \& Vortex diode 


\section{2}

Identification of inception of a cavitation is an important design parameter. The measured cavitation inception conditions are presented in Figure 9 for the Venturi, orifice and vortex units, along with the numerical predictions of the cavitation inception point. For the Venturi, evidence of cavitation is first observed just downstream of the throat, in the form of a thin ring of cavities which extend a short distance into the diffuser. This consistently first appears at a pressure drop across the device of 55 $\mathrm{kPa}$. CFD predictions indicate that the minimum pressure in the throat reaches vapour pressure slightly earlier at $51 \mathrm{kPa}$, which is in close agreement with observations. During testing the appearance of this initial cavitating region was accompanied by the obvious onset of a harsh noise, sounding like small stones being forced through the device. For the orifice, inception is first observed as a similar thin ring of cavities which initiates around the edge of the orifice restriction, extending a short distance into the throat. Inception is observed at a higher pressure ratio than that for the Venturi, at $85 \mathrm{kPa}$. CFD predictions again show reasonably good agreement, with vapour pressure predicted to be reached at this inlet edge at a pressure drop of $79 \mathrm{kPa}$. Again it was observed during testing that the first occurrence of cavitation is accompanied by the obvious introduction of an additional source of noise, although in the case of the orifice this was perceptibly lower in magnitude than that observed for the Venturi. Finally, Figure $9 \mathrm{c}$ presents the observed and predicted inception conditions for the vortex diode device. Inception was first observed as a thin line vortex initiating at the rear wall of the diode chamber. The close up image in Figure 9 c reveals some interesting details of the nature of the vortex core; a distinct phase separation is observed, with a filled gas core at the centre of the device, around which the camera images highlight smaller discrete cavities orbiting this central core. Whereas in the case of the linear flow devices the predicted inception conditions are both at lower pressure drops than those recorded by visual observation, the opposite is true for the vortex device; predictions indicate inception at $86 \mathrm{kPa}$, whereas during testing a distinct bubbly core was observed to form significantly earlier at a pressure drop of $50 \mathrm{kPa}$. This is accompanied by a perceptible change in noise level, however this is audibly lower than that observed for both of the linear flow devices. The appearance of a gas core below the expected cavitation conditions has been reported elsewhere, and this early inception may be attributed at least in part to non-condensable gas $57,58,65$.
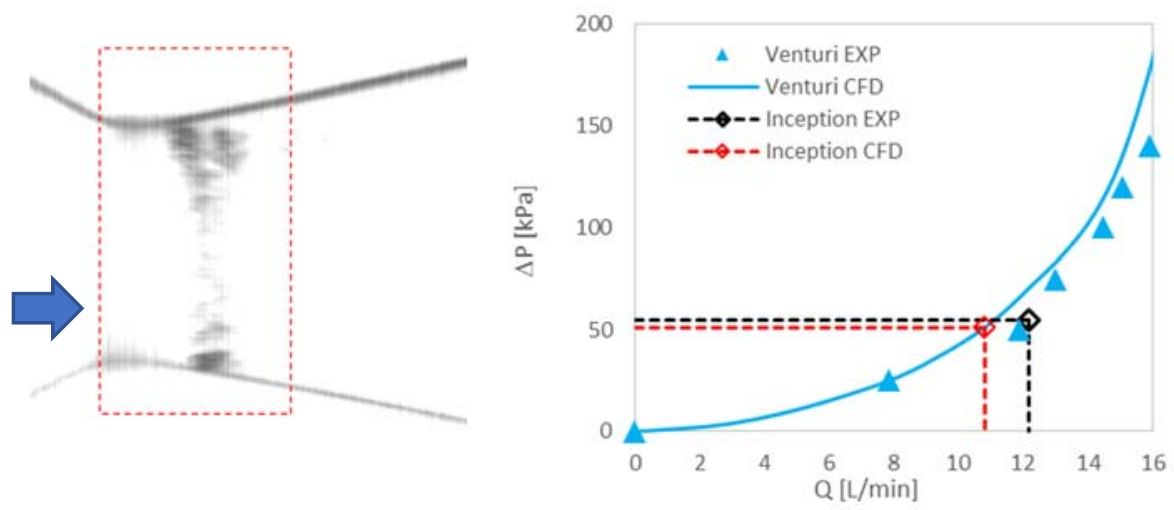

(a) Cavitation inception: Venturi (without swirl) 

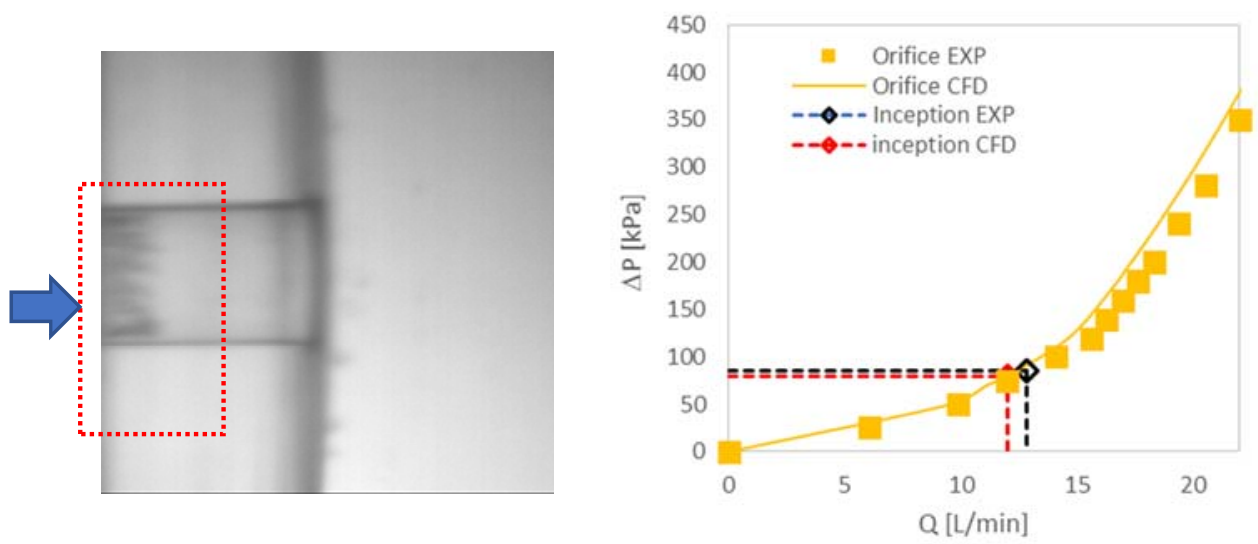

(b) Cavitation inception: Orifice (without swirl)
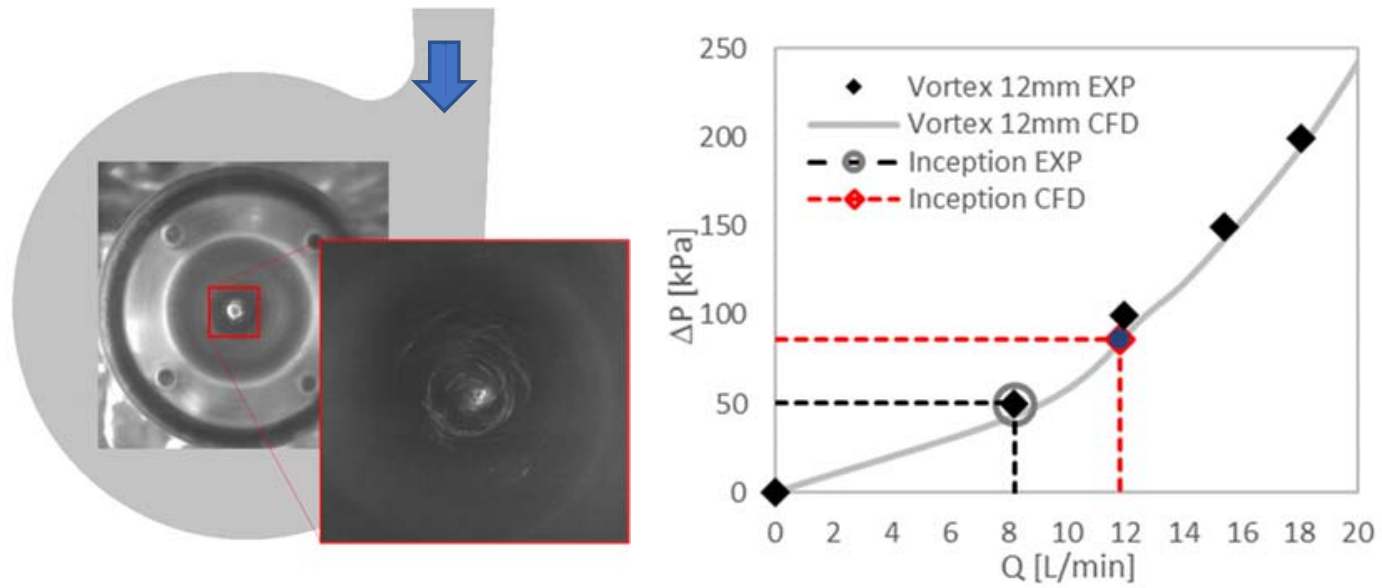

(c) Cavitation inception: Vortex diode

Figure 9: Cavitation inception; predicted \& experimentally observed

With swirl imposed for the orifice the inception pressure drop is recorded to reduce from $85 \mathrm{kPa}$ to $75 \mathrm{kPa}$. Similarly for the Venturi inception is observed to reduce from $55 \mathrm{kPa}$ to $50 \mathrm{kPa}$ when swirl is imposed. The corresponding predicted and experimentally observed cavitation inception points for the orifice and Venturi with swirl can be found in the supplementary information (Figure SI.2). Although the energy inputs required to initiate cavitation do not change significantly with the additional of the swirler, significant differences were observed in the nature and position of the cavitating regions for each device; Figure 10a presents a series of sequential frames for the orifice with swirl at inception, along with a collated time-average image. Time averaged CFD are also presented for comparison; in this case the legend colour bars directly relate to the computed phase volume fractions. The variations in grey intensity scale in the image data indicate the phase boundaries, with darker regions therefore highlighting the presence of large populations of smaller cavities; nonetheless, comparing the computed and experimentally recorded flow fields show good agreement between the position and extent of the cavitating flow regions. The introduction of swirl is shown to produce a cavitation zone along the central axis, where a thin, cavitating line vortex is formed. For the orifice, the images also indicate that edge cavitation is still present. The corresponding plots at inception for the Venturi (Figure 10b) show a similar situation; again a short line vortex is apparent, which is transient in nature, formed from the growth and collapse of discrete cavities. Some evidence still exists of edge cavitation at the Venturi throat surfaces, and this is apparently asymmetric as well as unsteady. 


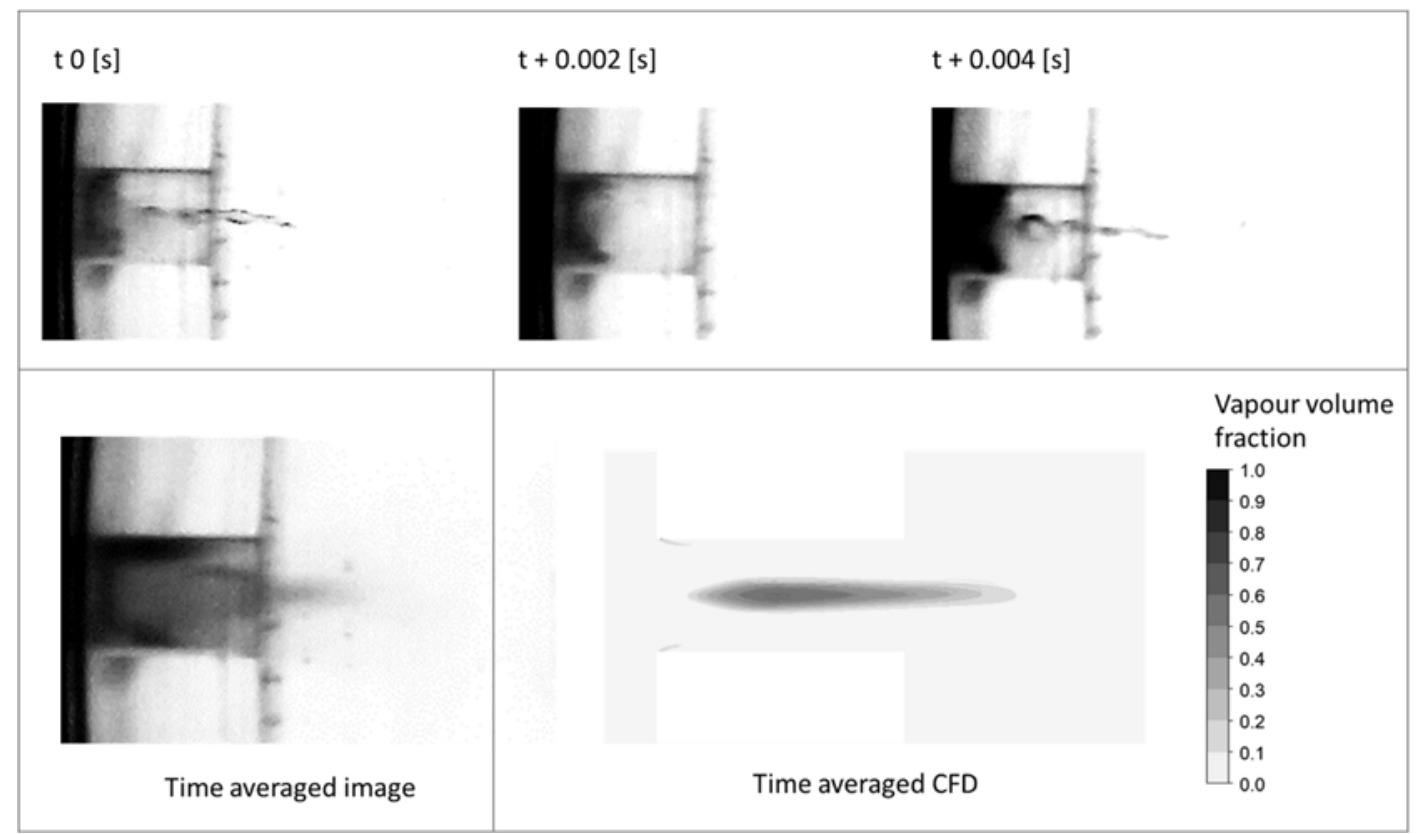

(a) Orifice

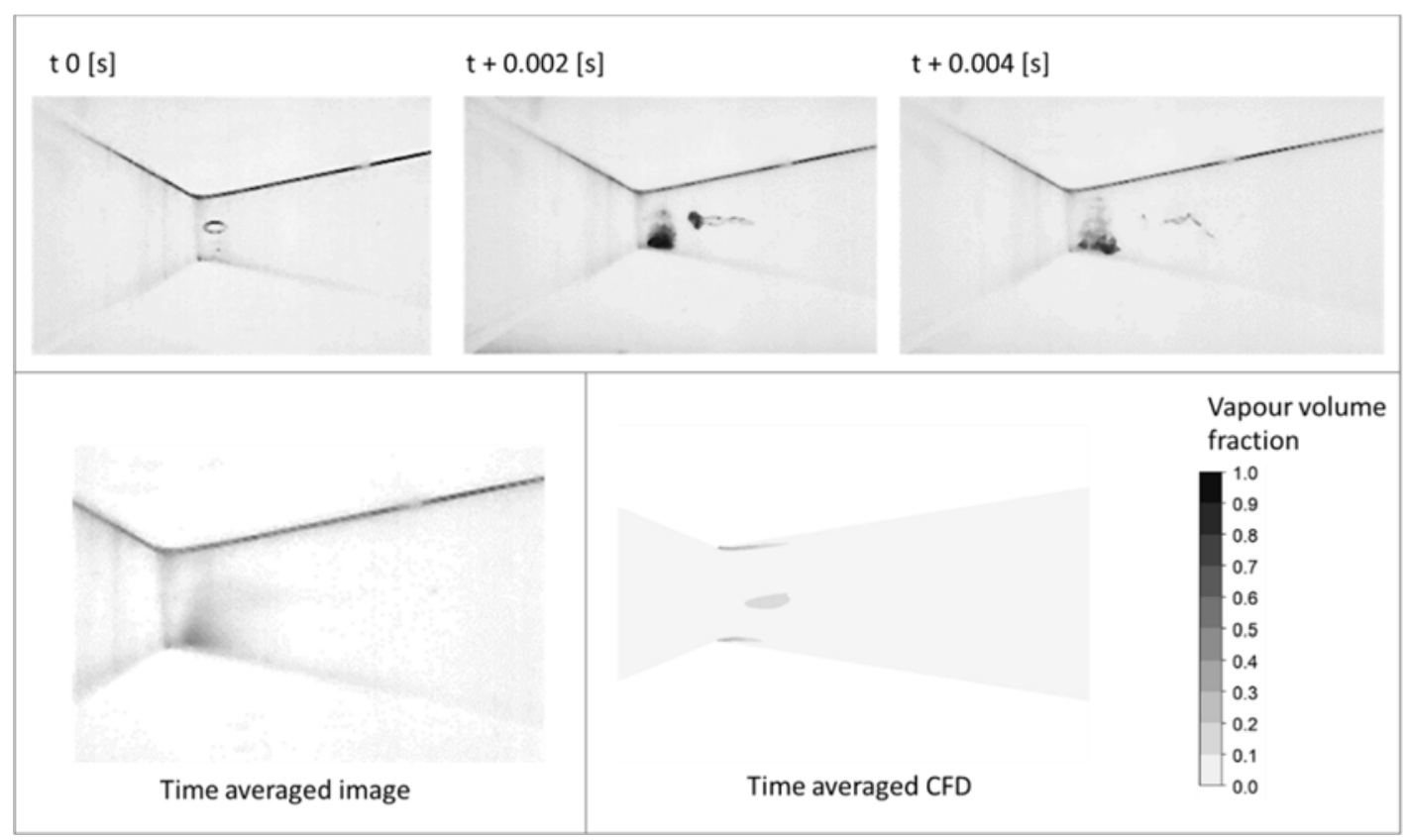

(b) Venturi

Figure 10: Cavitation inception for linear flow devices with swirl; recorded images \& CFD

Cavitation inception is highly dependent on the details of the local flow gradients within the device; Figure 11 shows the velocity profiles and corresponding pressure gradients in the throat of the Orifice. The plots reveal the influence of local accelerations in producing regions of low static pressure, at which cavitation is both visually observed and predicted to initiate. In the case of the orifice without swirl a low pressure region is formed as flow accelerates around the sharp edge of the throat; and as captured by the high speed images cavities therefore tend to form around this sharp edge. With the addition of swirl however the predictions reveal a pronounced shift in velocity gradient, with the highest velocities shown to move towards the axis. The lowest predicted static pressure regions are correspondingly found along the axis as opposed to the edge of the throat, and as such the cavitating structures begin to move in away from the solid surfaces. 


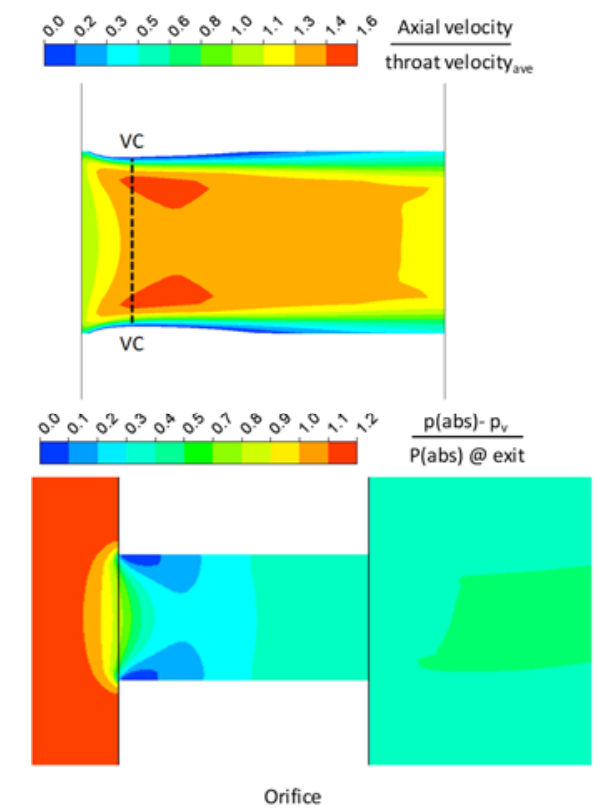

(a) Orifice; linear flow

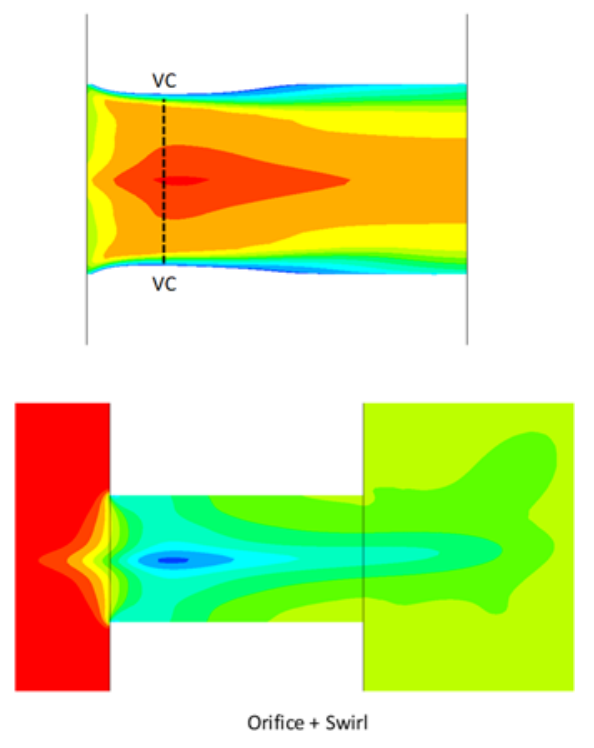

(b) Orifice, with swirler

Figure 11: Predicted Orifice velocity \& pressure gradients at inception

Cavitation inception is typically estimated by using the cavitation inception number, $\sigma_{i}$, which is defined as (with $\mathrm{C}=1$ ):

$$
\sigma_{i}=\frac{p_{d}-p_{v}}{1 / 2 \rho_{l}\left(C u_{i}\right)^{2}}
$$

Where $u_{i}$ is the characteristic velocity at inception, and $\mathrm{C}$ is a velocity correction factor. As cavitation inception was found to be highly sensitive to the precise details of the geometry and velocity gradients within each device, it is important to select appropriate characteristic velocity while calculating Cavitation number, $\sigma_{i}$. The parameter ' $C$ ' is introduced in Equation (9) for this purpose. If $\sigma_{i}$ is calculated simply based on the average throat velocity ( $u_{t}$ ave), the inception was found to occur at values of $\sigma_{i}$ higher than one (see Table 1) for most of the devices except Venturi. In order to define a more meaningful value for the characteristic velocity, the CFD simulations were interrogated in order to determine the size and position of the vena contracta in each instance. As indicated in Figure 11, for the orifice geometries an iso-surface was created to include axial velocity values greater than one, which reveals the shape of the vena contracta. The minimum radius along the perimeter of this surface was then calculated, and a sample plane created on which to calculate a mass averaged value of the local velocity. This same exercise was repeated for the Venturi geometries. For the vortex diode, as cavitation inception is driven by high swirl as opposed to linear acceleration, a different criterion is required to define the characteristic velocity used to calculate the cavitation number. The vortex diode produces a maximum swirl ratio in the centre of the device of $6 u_{t}$, and this ratio is found to be consistent across the range of flow rates considered in the present study. The maximum tangential velocity was therefore used as the characteristic velocity in the $\sigma_{i}$ calculations for the vortex diode. The resulting cavitation numbers are listed in Table 1 for each device, showing inception values to consistently approach values between $0.9-1.0$. 
Table 1: Cavitation numbers at the inception

$\begin{array}{rccc} & \sigma_{i} \mid u_{\mathrm{tave}} & \sigma_{\mathrm{i}} \mid \mathrm{u}_{\mathrm{CFD}} & \mathrm{C} \\ \text { Orifice } & 1.575 & 0.956 & 1.36 \\ \text { Orifice + Swirl } & 1.502 & 0.984 & 1.26 \\ \text { Venturi } & 1.007 & 0.998 & 1.00 \\ \text { Venturi + Swirl } & 1.235 & 0.952 & 1.10 \\ \text { Diode } & \mathrm{NA} & 0.922 & 6.00\end{array}$

The correction factors determined for the present range of geometries are listed in Table 1. For the orifice geometries, this correction factor is a function of the vena-contracta area. For the sharp-edged geometry studied here, the ratio of vena-contracta to throat area, $A_{v c} / A_{t}$ equates to 0.74 , which results in a required correction factor on the throat velocity of 1.36. For the vortex diode, the correction factor must be based on the maximum swirl ratio. In conclusion, the Cavitation number calculation must take account of the local velocity gradients and device losses in order to be a reliable indicator of cavitation inception.

\subsection{Flow characteristics: Linear flow devices}

Moving beyond inception conditions, the evolution of cavitation in the orifice with increasing pressure drop is presented in Figures 12 - 13 for the orifice and Venturi for purely axial inlet flow. Figure 12a presents image data for the orifice at a series of increasing pressure drops, showing both individual successive frames and a time average of the full recorded data set in each instance. Just beyond inception at $100 \mathrm{kPa}$, cavitation remains restricted to a short length after the sharp edge hole, and the images reveal the size and position of the vapour pocket to be relatively steady state. Beyond $200 \mathrm{kPa}$ however the cavitation region reaches the orifice exit, after which the vapour region breaks off under turbulence into a succession of smaller ring-cloud like structures, and ultimately into a fine cloud of bubbles. The CFD plots presented in Figure12b show good agreement with the high speed images; cavitation is predicted to initiate at the stagnation point after the sharp edge, and then shown to proceed along and eventually beyond the throat as pressure ratio increases. While the complex details of cavity break up are beyond the capability of the present homogenous fluid model, the time averaged vapour fraction plots nonetheless successfully reproduce the time averaged experimental data in terms of the position and extent of the cavitation region.

Figure 13a provides corresponding experimental and predicted two-phase behaviour for the linear flow Venturi. The static images highlight the inherently transient nature of the cavitation behaviour; the vapour pocket formed at the edge of the throat periodically advances along the diffuser for a short distance before collapsing into a cloud of bubbles, upon which it retreats to the throat to start the process again. As pressure ratio increases, the distance travelled from the throat into the diffuser by the relatively coherent vapour pocket increases, and the images show that some liquid is shown to flow back along the diffuser wall (the often termed "re-entrant jet" effect). This relatively coherent vapour pocket then again breaks up into larger cloud cavity structures, which themselves progressively break down into a cloud of fine dispersed cavities. Comparison of these images with the CFD predictions in Figure13b again reveals good agreement with time averaged cavitation behaviour. Whereas again the details of the cavitation cloud shedding behaviour are not captured by the homogeneous fluid approach, the CFD results do clearly capture the transient, periodic advance and retreat of the cavitation region along the diffuser. Further details of the transient behaviour and observed dominant frequencies are provided in the subsequent section. 


\subsection{Flow characteristics: Swirling flow devices}

The influence of swirl on the cavitation patterns in the Venturi and orifice is clearly shown in Figure 14a which provides a direct comparison of the swirling Venturi, orifice and diode. Firstly the orifice with swirl exhibits a well-defined cavitating vapour core in the throat, which collapses and again breaks down into a succession of cavity cloud clusters downstream of the throat. The images also highlight some precession of the tail of the vortex core around the exit of the orifice. Again, some degree of edge cavitation also remains present. For the Venturi, the imposition of swirl has moved the cavitation zone into the centre of the device; a coherent vapour core can be seen in the middle of the throat, beyond which a large cavitation cloud extends roughly half way into the diffuser. While this is inherently unsteady, the addition of swirl acts to dampen the axial advance and retreat of the cavitation region exhibited in linear flow, and the cavity length remains relatively fixed. In comparison, in the vortex diode the images show a cavitating vortex core in the centre of the device which extends to the end of the axial port; at this location the sharp increase in cross section area causes the formation of a large cavitation cloud. The vortex core also exhibits periodic, precession movement. Corresponding flow visualization comparisons compiled at pressure drops of $100 \mathrm{kPa}$ and $300 \mathrm{kPa}$ are available in the supplementary information (Figures SI.3 and SI.4). An accompanying comparison of the CFD predicted flow fields is given in Figure14, which provides further insight into the hydrodynamic characteristics of each device. In each case the predictions capture the effect of swirl in moving the cavitation zone towards the axis; additionally, for the Venturi and orifice configurations some edge cavitation effects are also predicted, which are also revealed by the camera images. For the vortex diode the predictions successfully capture the cavitating vortex core, which is shown to extend to the end of the axial port where it condenses back at the point of cross section increase. The CFD results also reveal a further prominent feature of the vortex diode; a reverse flow core produced by the high swirl ratio. This velocity deficit region is predicted to extend up to 40 diameters downstream, and this is also confirmed by visual observations in the transparent downstream tubing of the test rig. A further complimentary set of CFD predicted flow field plots at different operating is also found in the supplementary information, Figures SI.6 to SI.8. 

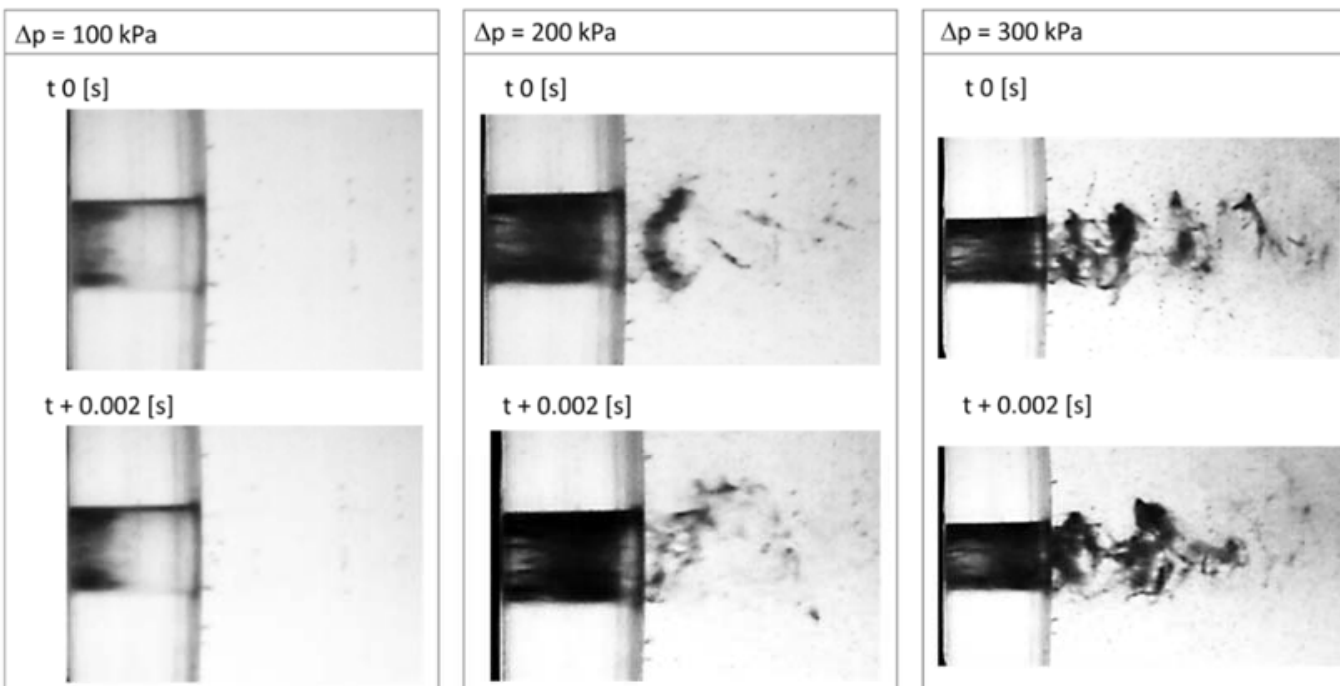

$\mathrm{t}+0.002[\mathrm{~s}]$

$t+0.004[s]$
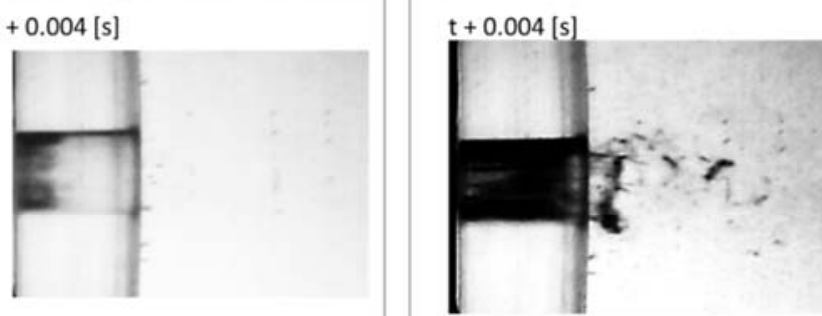

Time average:

Time average:
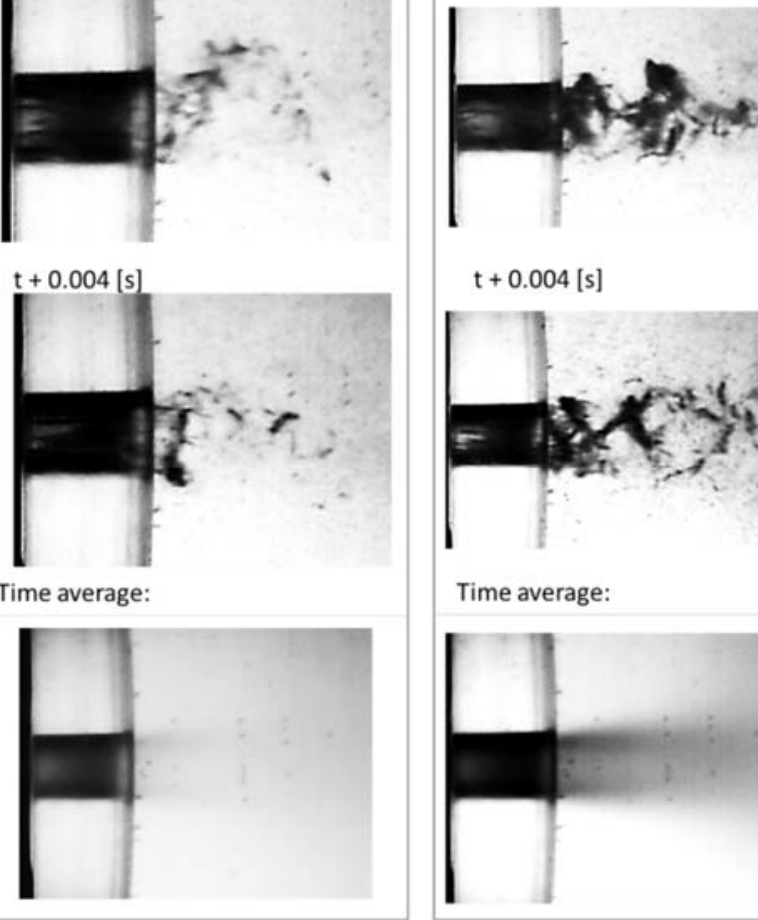

$t+0.004[s]$

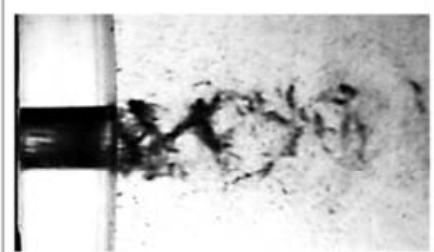

Time average:

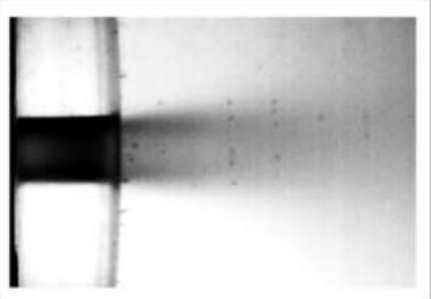

(a) High speed flow visualization of cavitation evolution with $\Delta \mathrm{p}$
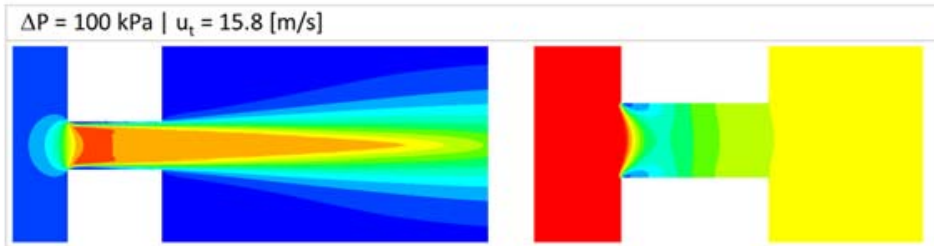

$\Delta P=300 \mathrm{kPa} \mid \mathrm{u}_{\mathrm{t}}=27.3[\mathrm{~m} / \mathrm{s}]$
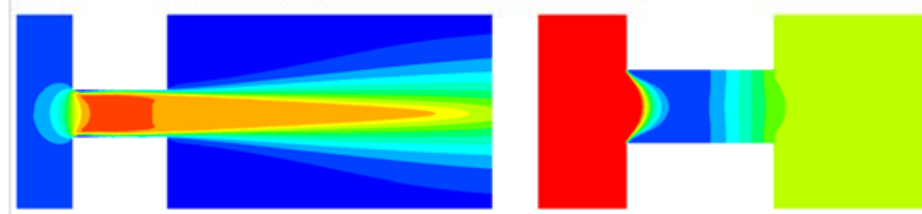

Axial velocity $\left(u_{x} / u_{t}\right)$

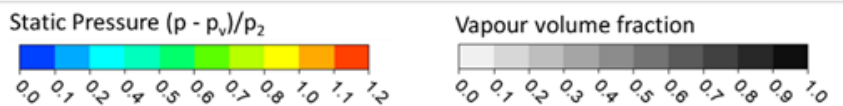

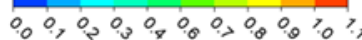

(b) Predicted flow fields and cavitation extent

Figure 12: Key flow characteristics of linear flow orifice 


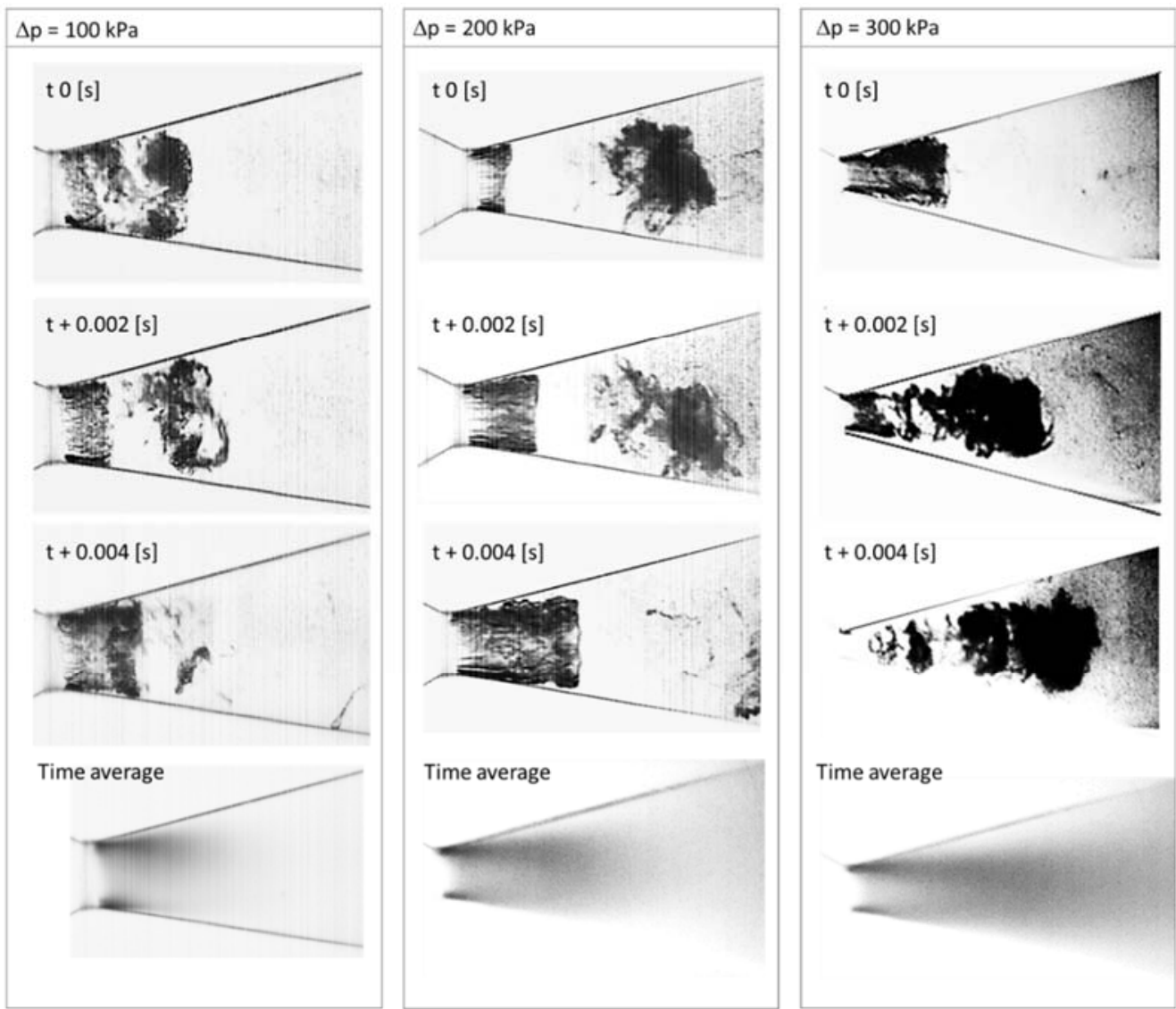

(a) High speed flow visualization cavitation evolution with $\Delta p$

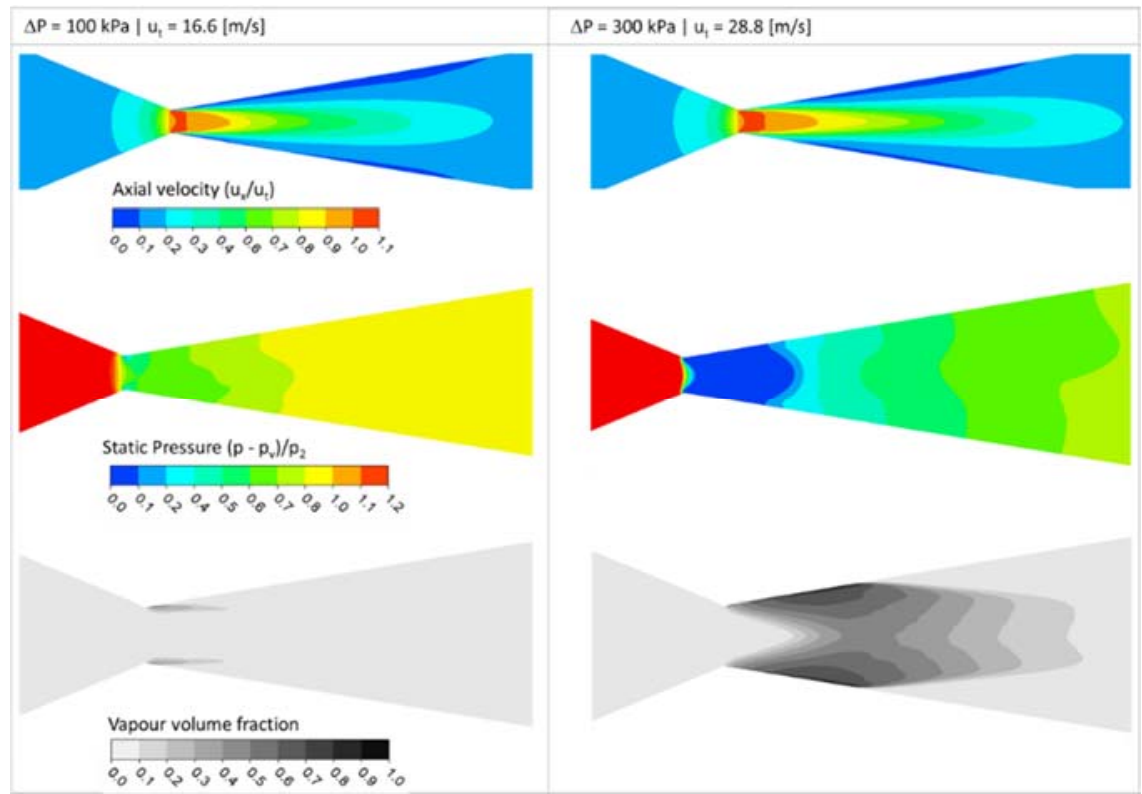

(b) Predicted flow fields and cavitation extent

Figure 13: Key flow characteristics of linear flow Venturi 

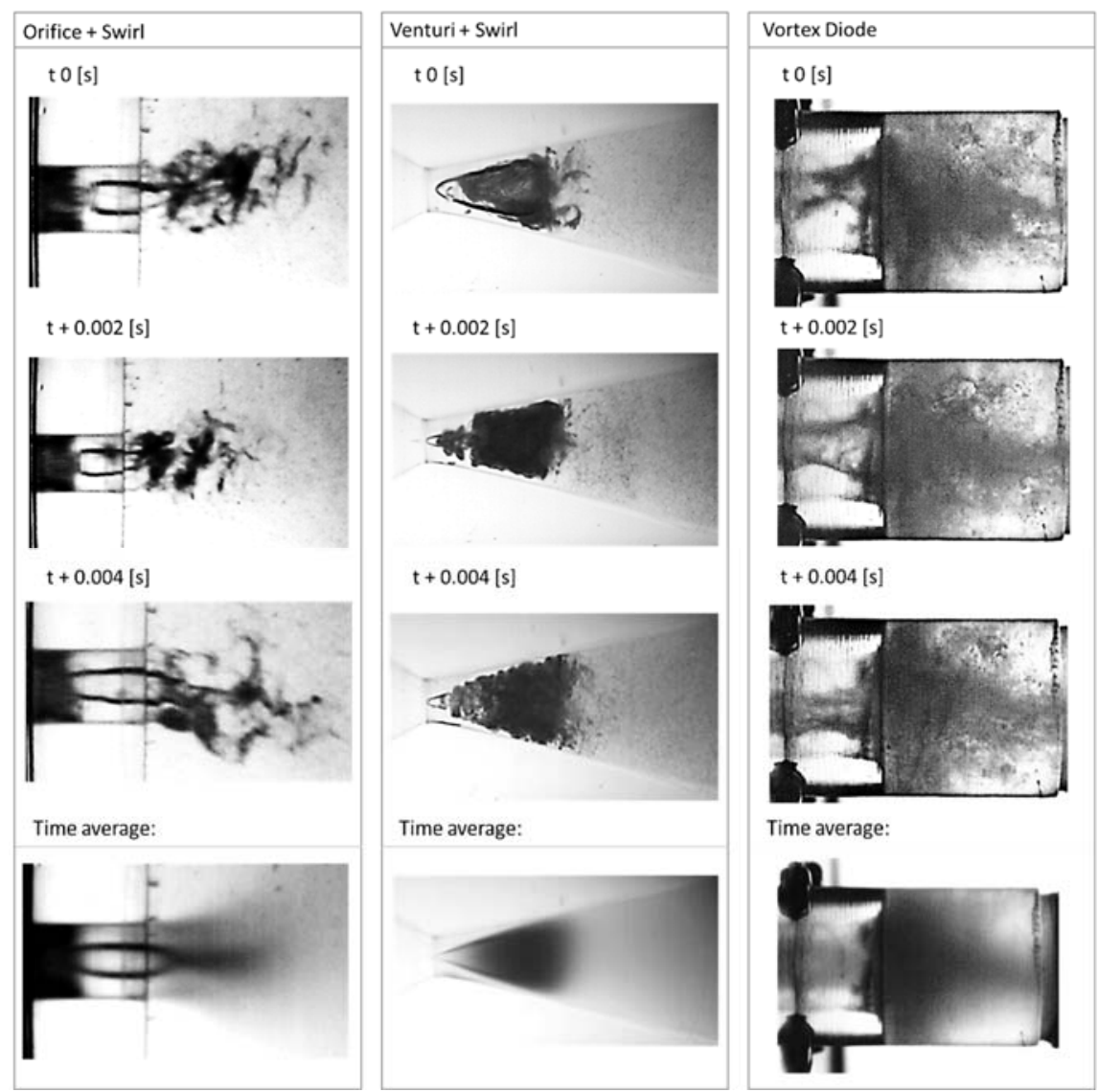

(a) High speed flow visualization; $\Delta p=200 \mathrm{kPa}$
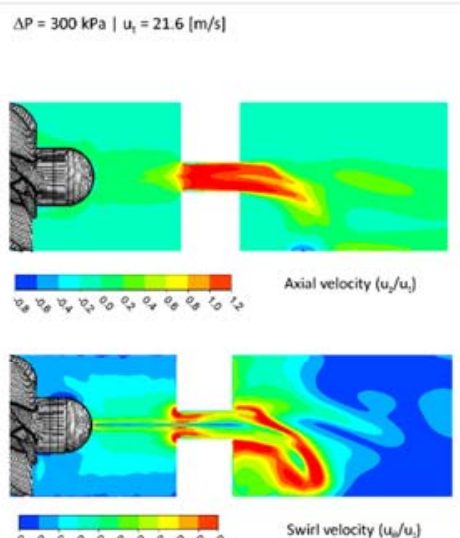

$\Delta P=300 \mathrm{kPa} \mid \mathrm{u}_{\mathrm{t}}=29.5[\mathrm{~m} / \mathrm{s}]$

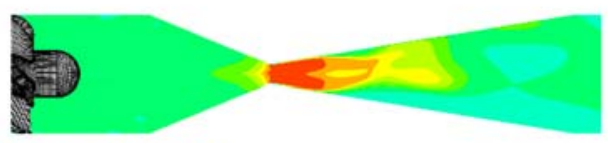

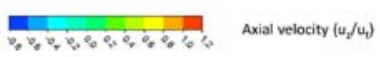

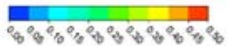

Swirl velocity $(u / u)$

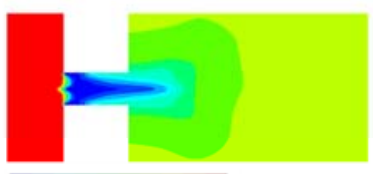

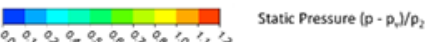
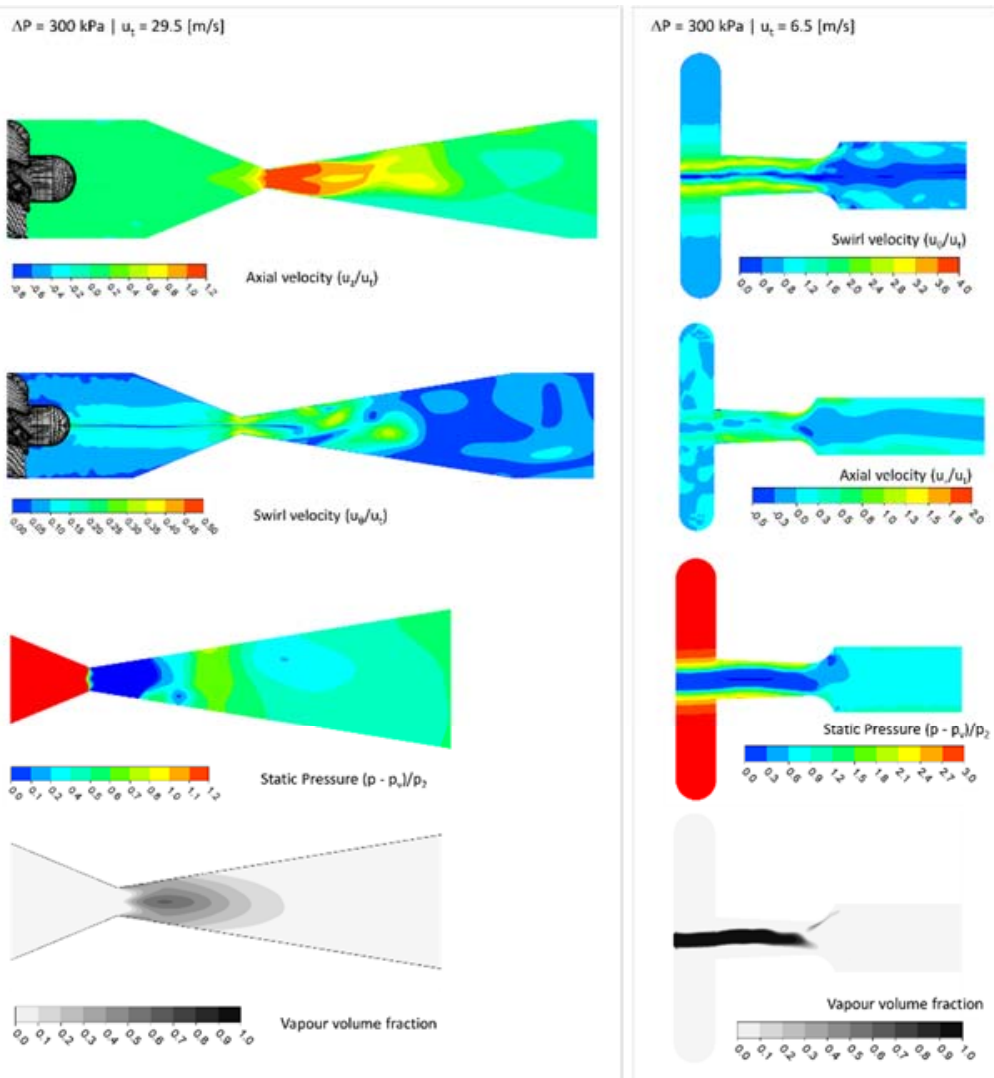

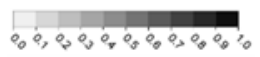
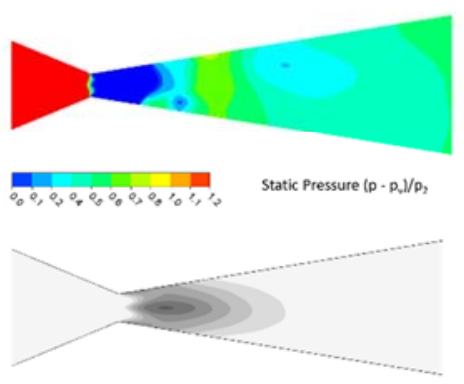

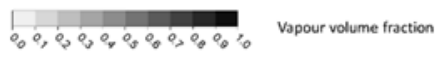

$.0,0^{\circ}, 0^{2}, 0^{\circ} 0^{\circ} 0^{2} 0^{\circ} 0^{\circ}$,

(b) CFD simulations

Figure 14: Comparison of flow characteristics of devices based on swirling flows 
The instantaneous images in the preceding section show transient shedding of the cavitation cloud downstream of the both the Venturi throat and orifice exit. In order to quantify this transient behaviour, the camera recordings were processed frame by frame so as to identify the dominant transient frequencies. Figure 15a shows the processing steps carried out; firstly the colour thresholds for each data set were processed frame by frame so as to clearly delineate between the secondary vapour phase and continuous liquid phase. Subsequently the data was interrogated to determine the minimum and maximum cavity lengths, and an area region defined which extended between these two limits. The average grey intensity within this area region was then calculated frame by frame, which produced a transient output signal for further processing. This process was repeated across a range of operating conditions in order to reveal any trends in oscillation frequency with flow rate; the resulting FFT plots for the Venturi are presented in Figure 15a. For the linear Venturi, the processed FFT plots revealed clearly defined single peaks, particularly towards the upper end of the flow rate and pressure drop range, reflecting the advance, retreat and shedding of the vapour cloud.

The dominant oscillation frequencies for the Venturi with and without swirl are compared In Figure $15 \mathrm{~b}$, and show distinctly different trends; the Venturi exhibits an increase in shedding frequency as pressure drop increases, whereas with inlet swirl imposed the dominant oscillation frequency is found to reduce beyond a pressure drop of $150 \mathrm{kPa}$. This is reflected in the images captured in Figure 15a, which show the effect that swirl has on stabilizing the variation in the length of the cavitation zone. The vortex diode results show clearly defined frequency peaks as a result of the precession movement of the vortex core. Surprisingly, the frequency is shown to follow a definite decrease with increasing flow rate, suggesting that the increase in swirl velocities acts to stabilise the precession movement of the cavitating core. The collapse of the core in the axial port, and the formation and breakup of the subsequent bubble cloud downstream is however highly stochastic, with frequencies higher than the capability of the camera system to resolve. The same is true for the process of cloud collapse into smaller structures and dispersed cavities for the Venturi and orifice configurations. The orifice with linear flow was also found to show an increase in shedding frequency with increasing flow rate, however with swirl imposed the processed camera data resulted in an essentially broadband noise signal, and no dominant frequencies could be found. Although precession of the vortex core is evident in the video recordings and images shown in Figure 15a this precession was low frequency (less than $10 \mathrm{~Hz}$ ) and essentially stochastic. It should be noted however that while no prominent periodic oscillations in the flow field were observed for the swirling orifice, it is also the case that the collapse of the cavitation cloud features a spectrum of complex, stochastic, high frequency collapse events, which cannot be resolved within the sampling limits of the current high speed camera system.

Comparison was also made between the dominant frequencies obtained by image processing and those obtained from the CFD data. The transient monitor point data just downstream of the throat of the linear Venturi at $200 \mathrm{kPa}$ is shown in Figure 15c. Subsequent FFT calculations yield an oscillation frequency of $200 \mathrm{~Hz}$ at $200 \mathrm{kPa}$, which is in excellent agreement with the experimental data. The same agreement could not be found when this exercise was repeated at $300 \mathrm{kPa}$ however; the CFD predictions indicate an increase in oscillation amplitude, accompanied by a significant reduction in frequency to $60 \mathrm{~Hz}$. As mentioned before, with this homogeneous fluid model approach the details of the shedding mechanism are not resolved, which may explain this discrepancy; the results will however prove useful in exploring alternative formulations of turbulence closure and multi-phase models in future work. It may be that scale resolving approaches, capable of capturing more of the complex interactions between turbulent flow structures and the corresponding phase interactions, may capture these specific transients more accurately. For the vortex diode, transient CFD monitored data is also shown in Figure 15c, and the predicted oscillations were found to successfully reproduce the key trend in the processed flow visualisation results; at $100 \mathrm{kPa}$ the vortex core precession 
frequency is predicted to be $340 \mathrm{~Hz}$, whereas at $300 \mathrm{kPa}$ this is predicted to reduce down to $35 \mathrm{~Hz}$, closely adhering to this same trend in the experimental data.
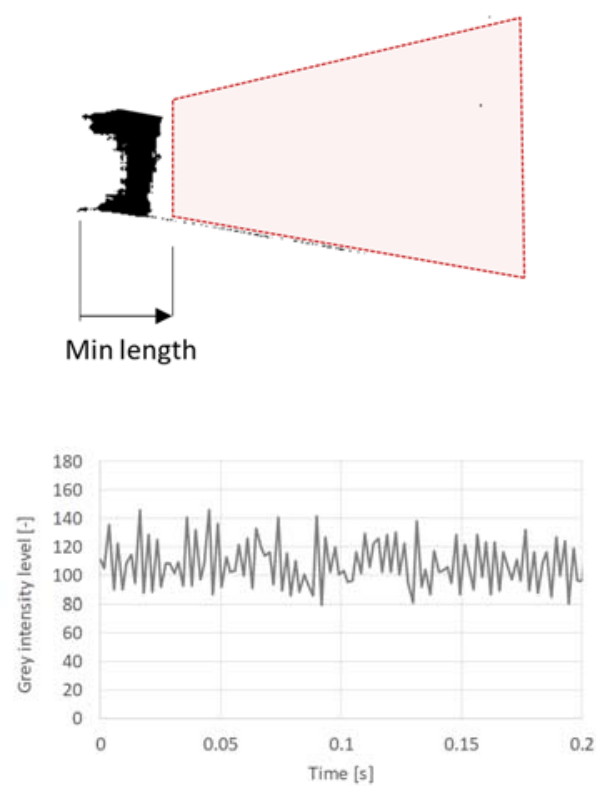
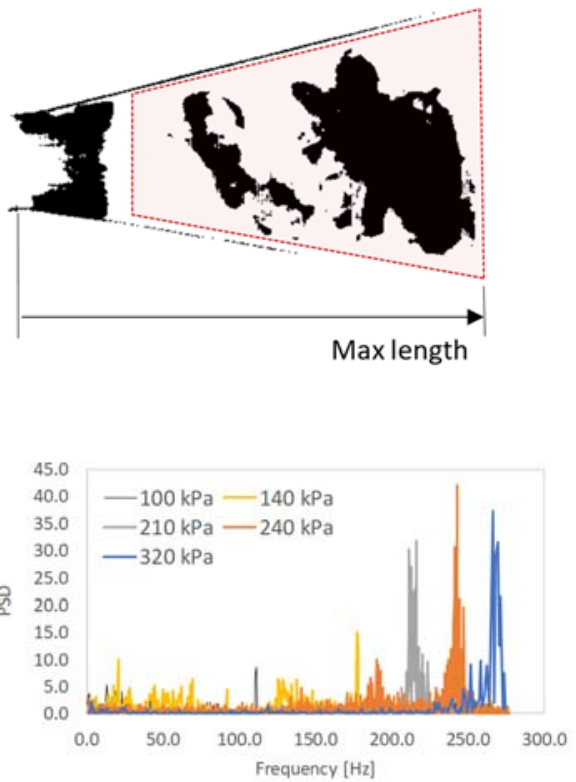

(a) Image processing for unsteady flow analysis

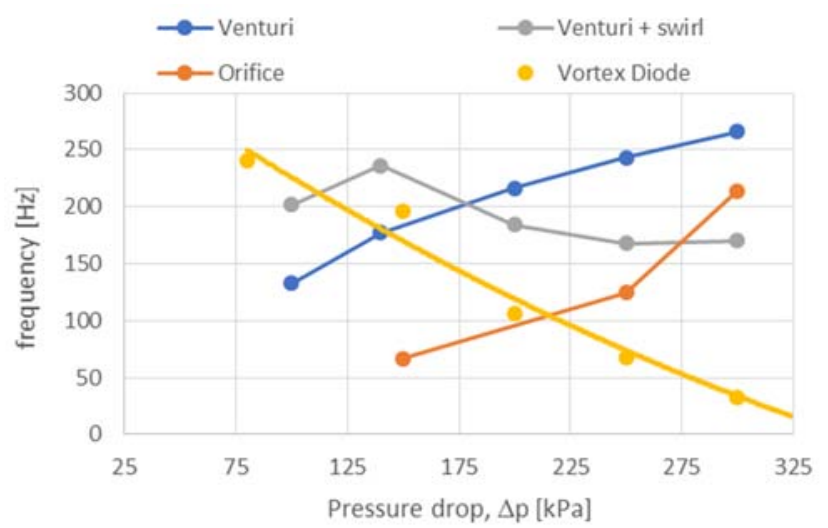

(b) Dominant oscillation frequencies

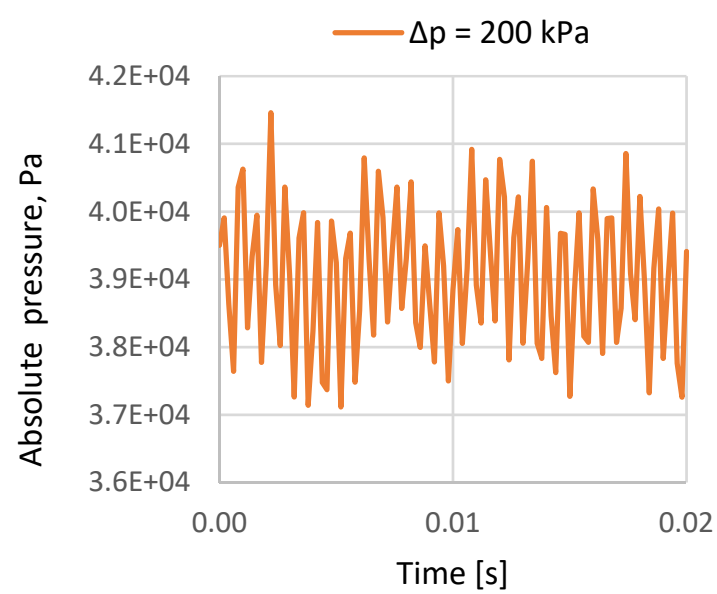

Linear venturi, $\Delta \mathrm{p}=200 \mathrm{kPa}$

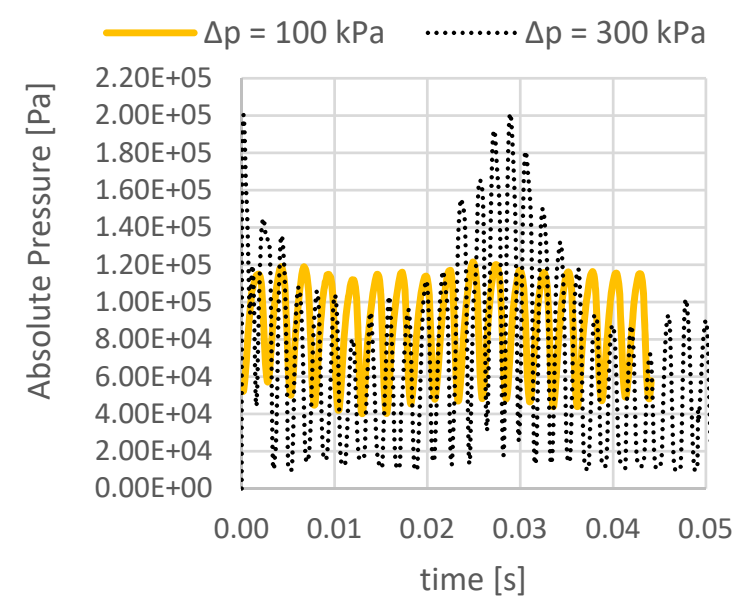

Vortex Diode, $\Delta \mathrm{p}=100 \mathrm{kPa} \& 300 \mathrm{kPa}$

(c) Computed pressure oscillations

Figure 15: Transient characteristics of flow generated by cavitation devices 
In order to fully explore the swirl parameter, the computational investigation was extended to study a wider range of inlet swirl ratios to the Venturi and orifice. Whereas the experimental swirler configuration achieved an inlet swirl ratio of the order of 2, this exercise allowed values of up to 16 to be simulated. Referring back to Figure 8, the experimental swirler configurations generated a lower measured pressure drop than the linear swirl cases; at this swirl ratio of 2 predictions for the orifice in particular shows a reduction in predicted turbulence production with the upstream swirler. A direct comparison of the predicted turbulence kinetic energy with and without swirl can be found in the supporting information, Figure SI.8. The predicted flow characteristic curves across the full range of swirl ratio are presented in Figure16a for the orifice and Venturi, which indicates that as swirl ratio is increased yet further beyond 2, the losses begin to exceed those of the linear flow devices. Results shown a consistent, monotonic shift to higher pressure drops for both devices from swirl ratios of 8 and above. For both the Venturi and orifice, the variation of turbulence fluctuations with increasing swirl number may explain the observed pressure loss versus flow rate characteristics: at swirl ratios of 2, there is a slight reduction in turbulence kinetic energy in comparison to the linear flow cases (see Figure SI.8). As the inlet swirl ratio is increased beyond this to values of the order of 6 , the turbulence kinetic energy was found to increase, reflected in the increase in pressure loss predictions with increasing swirl ratio. It is interesting to note that normalized values of turbulent kinetic energy yield consistent values across all 3 devices. The resulting evolution of the predicted flow fields with increasing swirl ratio are presented for the orifice and Venturi in in Figure 16a and Figure16b respectively. The contour plots highlight two key features; firstly, looking at the predicted vapour volume fractions for each device, inlet swirl ratios of the order of 6 and above are shown to produce a single, distinct cavitation zone in the centre of the device. Secondly, both sets of results show that as the maximum swirl ratio in the throat approaches and exceeds unity, a reverse flow "suction" region is shown to appear immediately downstream of the throat, replicating the behaviour of the vortex diode.

\subsection{Cavity trajectories}

The cavities generated in the low pressure region move with the fluid to the high pressure region and eventually collapse. The pressure (fluctuation) history experienced by the cavity determines conditions at the collapse and resulting physico-chemical effects (shear, localised temperature, pressure and generation of radicals). The turbulence fluctuations and frequency spectrum play an important role in the final collapse conditions of the individual generated cavities. While it is beyond the scope of the present investigation to explicitly link the hydrodynamic predictions to cavity dynamics calculations, the flow results will be useful in informing such calculations in future with realistic dynamic boundary conditions. It is therefore instructive to investigate the influence of swirl on cavity trajectories. In order to illustrate and compare the behaviour of discrete cavities as they move through the considered cavitation devices, a series of discrete phase model (DPM) calculations were performed on the converged continuous phase flow fields. Calculations were performed by initiating the trajectory calculations on the surface of the predicted vapour pocket, using an iso-surface with the vapour volume fraction set to 1.0. A fixed cavity size of $10 \mu \mathrm{m}$ was assumed for the trajectory calculations, with the pressure gradient and added mass terms accounted for in the force balance calculations owing to the large density difference. Example trajectory calculations are shown for each device configuration in Figure 17, again highlighting the significant influence that varying degrees of swirl have on the generated cavities. For the Venturi and diode, the in line swirler acts to force the generated cavities towards the axis, whereas the DPM trajectories for the vortex diode highlight how the cavities revolve tightly around the vortex core, before being dispersed in the relatively high turbulence region downstream of the axial port 


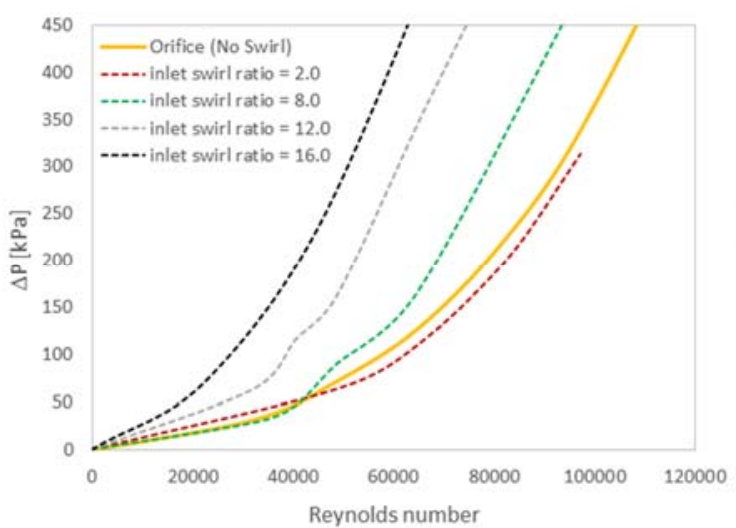

Orifice

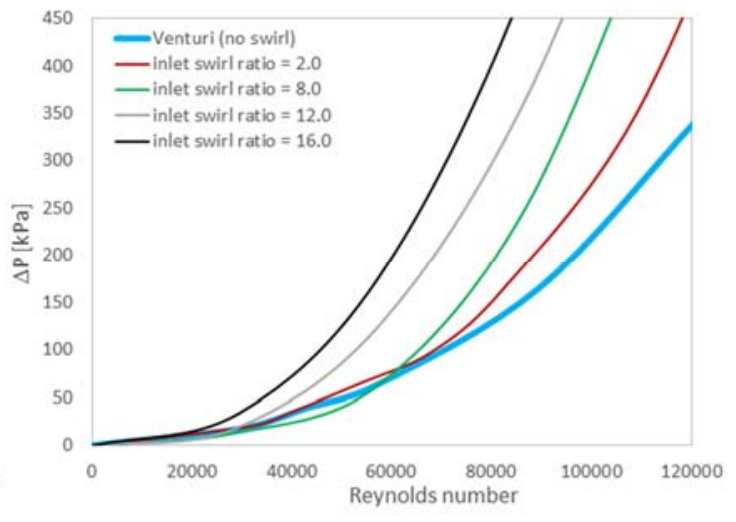

Venturi

(a) Pressure drop versus Reynolds number
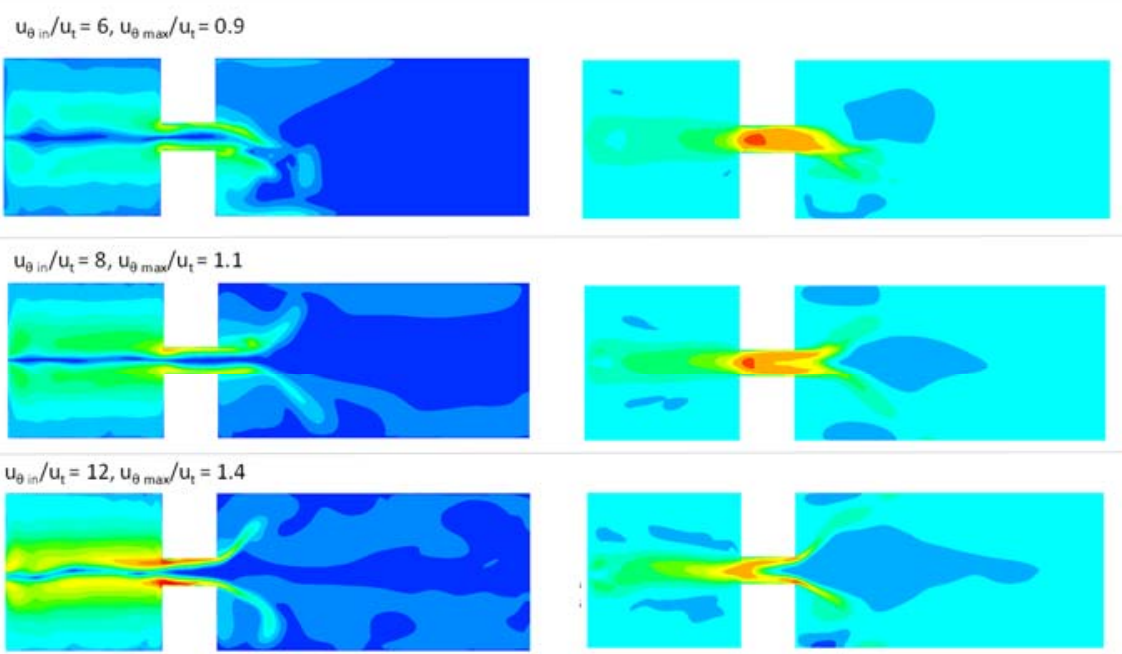

Lwirl velocity
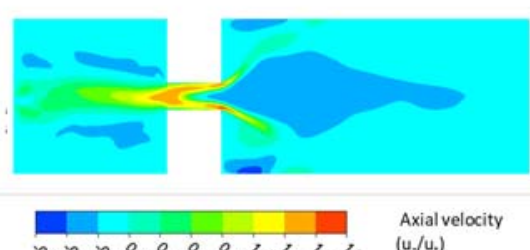

(b) CFD simulationsion

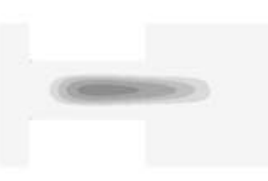

(b) CFD simulations: Orifice

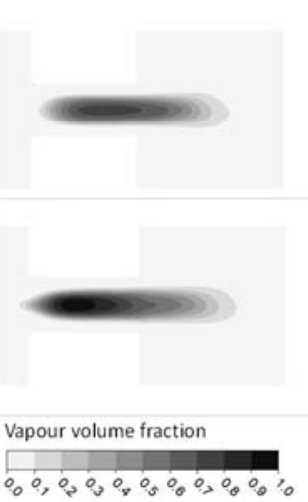

$\mathrm{u}_{\theta \mathrm{in}} / \mathrm{u}_{\mathrm{t}}=6, \mathrm{u}_{\theta \text { max }} / \mathrm{u}_{\mathrm{t}}=0.7$
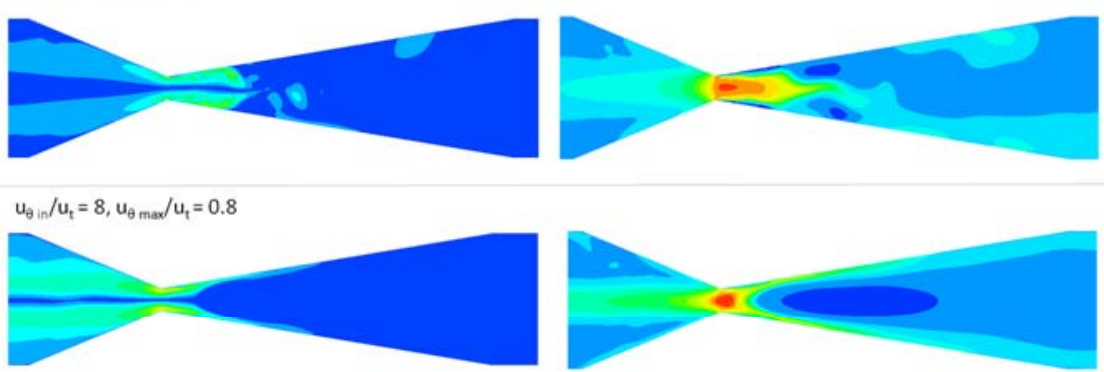

$\mathrm{u}_{\theta \mathrm{in}} / \mathrm{u}_{\mathrm{t}}=12, \mathrm{u}_{\theta \max } / \mathrm{u}_{\mathrm{t}}=1.2$
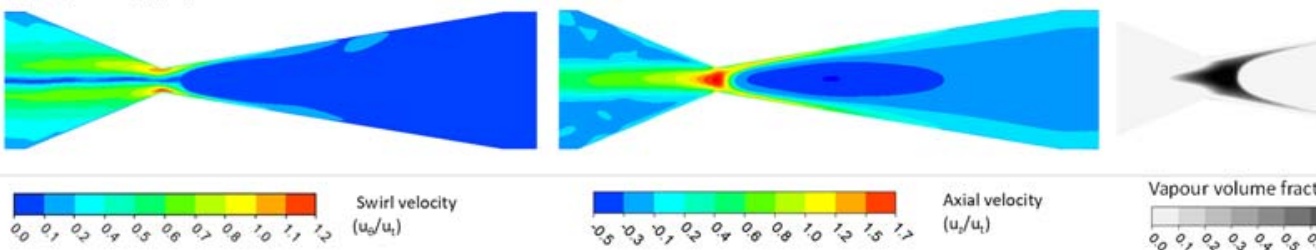

Vapour volume fraction

(c) CFD simulations: Venturi

Figure 16: Influence of high swirl ratios on flow characteristics of Orifice and Venturi 


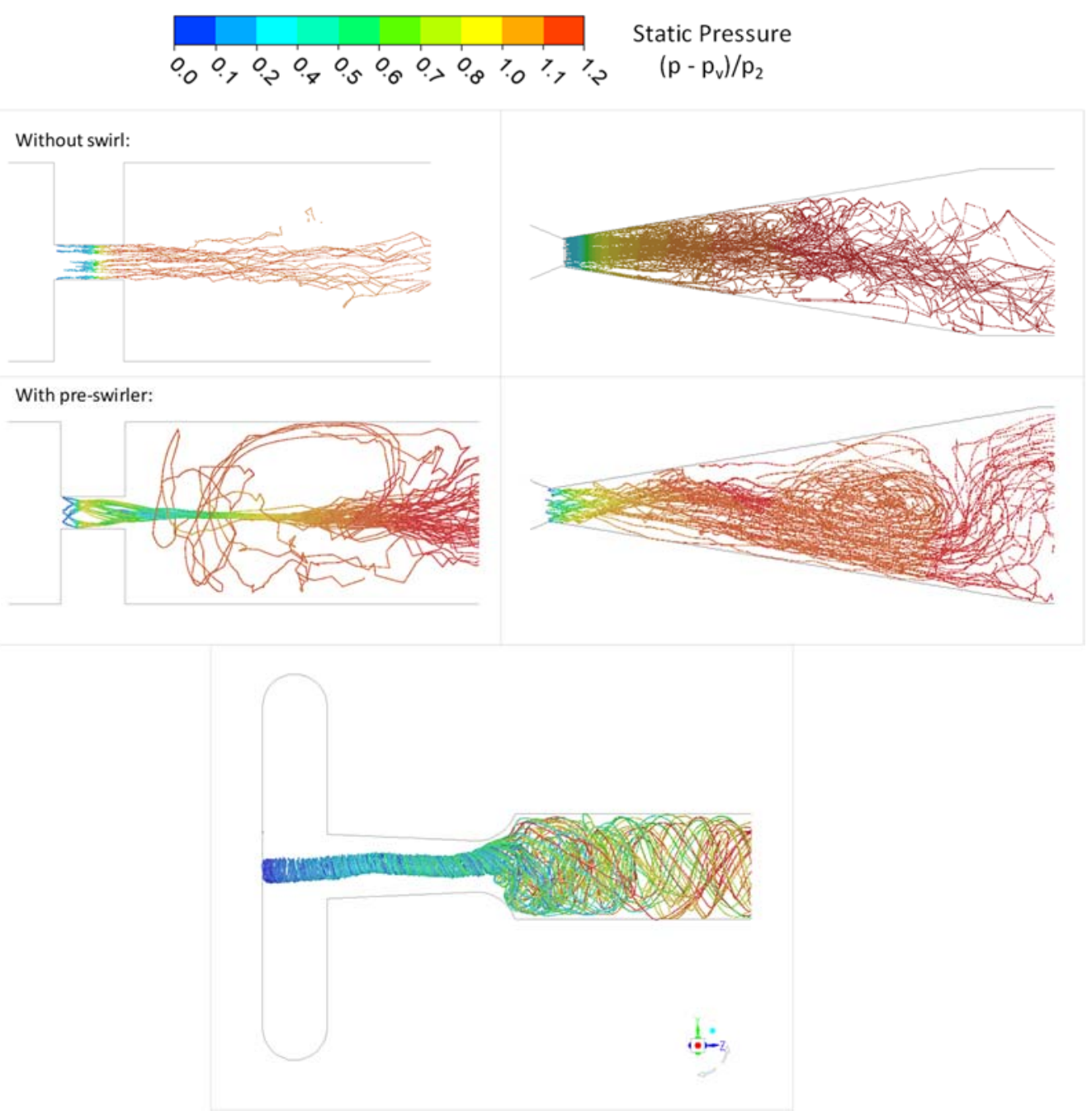

Figure 17: Discrete phase trajectories; Orifice \& Venturi with and without pre-swirler

\section{Conclusions \& future work}

The work presented in this paper for the first time presents a comprehensive comparison of cavitation behaviour between different configurations of commonly used cavitation devices. Multiphase CFD modelling has been successfully used to design a series of experimental test geometries which produce matched flow rate versus pressure drop data across a wide operating range, enabling direct comparisons to be drawn on the nature of the generated cavitation behaviour. The influence of key design parameters has been clearly identified. The key findings of the present investigation are summarized in the following:

- Results reveal that the vortex diode and swirling Venturi exhibit the lowest inception pressure drop of $50 \mathrm{kPa}$, with the orifice inception pressure significantly higher at $80 \mathrm{kPa}$. Calculation of cavitation inception number required careful definition of the characteristic velocity, informed by local flow gradients. When defining the cavitation number therefore, it is concluded that local accelerations and losses must be accounted for in order to use this parameter as a reliable indicator of cavitation inception between different devices.

- The influence of imposing swirl on Venturi and orifice geometries has been clearly captured; using an inline swirler the cavitating structures were successfully manipulated so as to move 
cavitation further towards the axis of the devices away from solid surfaces. This was achieved without any additional penalty in pressure losses.

- Parametric studies of swirl ratio revealed the trends in flow characteristics with increasing device inlet swirl; beyond swirl ratios of 2 increased pressure loss is incurred, and turbulence intensity increases.

- Sufficient increase in inlet swirl ratios of the order of 6 were found to produce a single cavitation zone at the device axis, with no cavitation shown at the surfaces of the throat.

- At high swirl ratios, when the ratio of tangential to axial velocities locally reach the order of unity, the vortex strength is sufficient to create a region of negative velocity suction flow downstream of the throat.

- Swirl is therefore a potentially useful design parameter in mitigating against cavitation erosion, as well as tuning the turbulence properties and cavitation characteristics of the device.

The insights provided by the high speed flow visualization data and detailed CFD models open up clear potential paths for future improved device designs. Alongside this work, a complimentary experimental programme is ongoing to evaluate the performance of each of the configurations presented here for wastewater treatment applications. The degradation performance results of these investigations will be published separately. The quantitative hydrodynamic data presented here, coupled with discrete cavity collapse predictions informed by realistic hydrodynamic boundary conditions, will offer further insight into the complex relationships between device cavitation characteristics and the resulting physio-chemical mechanisms produced. The initial development of a coupled multi-layer modelling approach has been published previouslys ${ }^{61}$, and these results will form a sound platform to further develop this approach, and to guide the optimised design of hydrodynamic cavitation devices by appropriately harnessing linear and swirling flows.

\section{Supporting information:}

The supplementary information has the following figures:

Figure SI.1: Vortex Diode mesh sensitivity

Figure SI.2: Predicted \& observed cavitation inception for swirl orifice \& venturi

Figure SI.3: High speed flow visualization; rotational flow comparison, ? $\mathrm{p}=100 \mathrm{kPa}$

Figure SI.4: High speed flow visualization; rotational flow comparison, ? $\mathrm{p}=300 \mathrm{kPa}$

Figure SI.5: Predicted flow field results, Venturi with swirler [? $\mathrm{p}=100 \mathrm{kPa}$, ?] $\mathrm{p}=300 \mathrm{kPa}$ ]

Figure SI.6: Predicted flow field results, Orifice with swirler [? $\mathrm{p}=100 \mathrm{kPa}$, ?p $=300 \mathrm{kPa}$ ]

Figure SI.7: Predicted flow field results, vortex diode [100 kPa, ? $\mathrm{p}=300 \mathrm{kPa}]$

Figure SI.8: Turbulent kinetic energy comparison with and without swirl

\section{Conflict of Interest:}

One of the authors (VVR) is a founder director of Vivira Process Technologies Pvt. Ltd. which commercially offers vortex based cavitation device used in this study.

\section{References}

1. Li S. Tiny bubbles challenge giant turbines: Three gorges puzzle. Interface Focus. 2015;5(5):1-25. doi:10.1098/rsfs.2015.0020

2. Rayleigh, Lord. VIII. On the pressure developed in a liquid during the collapse of a spherical cavity. Philos Mag Ser 6. 1917;34(200):94-98. doi:10.1080/14786440808635681

3. Keller JB, Miksis M. Bubble oscillations of large amplitude. 1980;68(2):628-633. 
4. Yasui K. Alternative model of single-bubble sonoluminescence. Am Soc Phys Rev. 1997;56(6):6750-6760.

5. Alehossein H, Qin Z. Numerical analysis of Rayleigh-Plesset equation for cavitating water jets. Int J Numer Meth Engng. 2007;72:780-807.

6. Qin Z, Alehossein H. Heat transfer during cavitation bubble collapse. App/ Therm Eng. 2016;105:10671075. doi:10.1016/j.applthermaleng.2016.01.049

7. Knapp RT, A.Hollander. Laboratory Investigations of the Mechanism of Cavitation. Trans ASME. 1948;(July):419-435.

8. Moholkar VS, Senthil Kumar P, Pandit AB. Hydrodynamic cavitation for sonochemical effects. Ultrason Sonochem. 1999;6(1-2):53-65. doi:10.1016/S1350-4177(98)00030-3

9. Suslick KS, Mdleleni MM, Ries JT. Chemistry induced by hydrodynamic cavitation. J Am Chem Soc. 1997;119(39):9303-9304. doi:10.1021/ja972171i

10. Plesset MS, Ellis AT. On the Mechanism of Cavitation Damage. Trans ASME. 1955;77(7):1055-1064. doi:10.1016/0043-1648(58)90042-5

11. Eisenberg P. Cavitation Damge, Hydronautics Inc. Technical Report 233-1.; 1963.

12. Hammitt FG. Damage to solids caused by cavitation. In: Royal Society Discussion on Deformation of Solids due to Liquid Impact; 1965.

13. Dular M, Bachert B, Stoffel B, Sirok B. Relationship between cavitation structures and cavitation damage. 2004;257:1176-1184. doi:10.1016/j.wear.2004.08.004

14. Sreedhar BK, Albert SK, Pandit AB, Albert SK, Pandit AB. Cavitation damage: theory and measurements a review. Wear. 2017;372-373:177-196. doi:10.1016/j.wear.2016.12.009

15. Gaikwad V, Ranade VV. Disinfection of Water Using Vortex Diode as Hydrodynamic Cavitation Reactor. Asian J Chem. 2016;28(8):1867-1870.

16. Capocelli M, Musmarra D, Prisciandaro M, Industriale I. Chemical Effect of Hydrodynamic Cavitation : Simulation and Experimental Comparison. 2014;00(00). doi:10.1002/aic

17. Mancuso G, Langone M, Andreottola G. A swirling jet-induced cavitation to increase activated sludge solubilisation and aerobic sludge biodegradability. Ultrason Sonochem. 2017;35:489-501. doi:10.1016/j.ultsonch.2016.11.006

18. Terán Hilares R, Ramos L, da Silva SS, Dragone G, Mussatto SI, Santos JC dos. Hydrodynamic cavitation as a strategy to enhance the efficiency of lignocellulosic biomass pretreatment. Crit Rev Biotechnol. 2018;38(4):483-493. doi:10.1080/07388551.2017.1369932

19. Nakashima K, Ebi Y, Shibasaki-Kitakawa N, Soyama H, Yonemoto T. Hydrodynamic Cavitation Reactor for Efficient Pretreatment of Lignocellulosic Biomass. Ind Eng Chem Res. 2016;55(7):1866-1871. doi:10.1021/acs.iecr.5b04375

20. Suryawanshi NB, Bhandari VM, Sorokhaibam LG, Ranade V V. A Non-catalytic Deep Desulphurization Process using Hydrodynamic Cavitation. Sci Rep. 2016;6(September):1-8. doi:10.1038/srep33021

21. Maddikeri GL, Gogate PR, Pandit AB. Intensified synthesis of biodiesel using hydrodynamic cavitation reactors based on the interesterification of waste cooking oil. Fuel. 2014;137:285-292. doi:10.1016/j.fuel.2014.08.013

22. Kelkar MA, Gogate PR, Pandit AB. Intensification of esterification of acids for synthesis of biodiesel using acoustic and hydrodynamic cavitation. Ultrason Sonochem. 2008;15(3):188-194. doi:10.1016/j.ultsonch.2007.04.003

23. Albanese L, Ciriminna R, Meneguzzo F, Pagliaro M. Beer-brewing powered by controlled hydrodynamic cavitation: Theory and real-scale experiments. J Clean Prod. 2017;142:1457-1470. doi:10.1016/j.jclepro.2016.11.162

24. Carpenter J, George S, Saharan VK. Low pressure hydrodynamic cavitating device for producing highly stable oil in water emulsion: Effect of geometry and cavitation number. Chem Eng Process Process Intensif. 2017;116(2016):97-104. doi:10.1016/j.cep.2017.02.013

25. Rajoriya S, Carpenter J, Saharan VK, Pandit AB. Hydrodynamic cavitation: An advanced oxidation process for the degradation of bio-refractory pollutants. Rev Chem Eng. 2016;32(4):379-411. doi:10.1515/revce2015-0075

26. Dular M, Griessler-Bulc T, Gutierrez-Aguirre I, et al. Use of hydrodynamic cavitation in (waste)water treatment. Ultrason Sonochem. 2016;29:577-588. doi:10.1016/j.ultsonch.2015.10.010

27. Ranade V V., Bhandari VM. Industrial Wastewater Treatment, Recycling, and Reuse-Past, Present and Future. Elsevier Ltd.; 2014. doi:10.1016/B978-0-08-099968-5.00014-3

28. Gogate PR, Mujumdar S, Thampi J, Wilhelm AM, Pandit AB. Destruction of phenol using sonochemical reactors: Scale up aspects and comparison of novel configuration with conventional reactors. Sep Purif Technol. 2004;34(1-3):25-34. doi:10.1016/S1383-5866(03)00171-0 
29. Capocelli M, Prisciandaro M, Lancia A, Musmarra D. Hydrodynamic cavitation of p-nitrophenol: A theoretical and experimental insight. Chem Eng J. 2014;254:1-8. doi:10.1016/j.cej.2014.05.102

30. Pradhan AA, Gogate PR. Removal of p-nitrophenol using hydrodynamic cavitation and Fenton chemistry at pilot scale operation. Chem Eng J. 2010;156(1):77-82. doi:10.1016/j.cej.2009.09.042

31. Sivakumar $M$, Pandit $A B$. Wastewater treatment : a novel energy efficient hydrodynamic cavitational technique. 2002;9:123-131.

32. Mancuso G. Experimental and numerical investigation on performance of a swirling jet reactor. Ultrason Sonochem. 2018;49(May):241-248. doi:10.1016/j.ultsonch.2018.08.011

33. Mancuso G, Langone M, Laezza M, Andreottola G. Decolourization of Rhodamine B: A swirling jetinduced cavitation combined with $\mathrm{NaOCl}$. Ultrason Sonochem. 2016;32:18-30. doi:10.1016/j.ultsonch.2016.01.040

34. Wang X, Wang J, Guo P, Guo W, Li G. Chemical effect of swirling jet-induced cavitation: Degradation of rhodamine $B$ in aqueous solution. Ultrason Sonochem. 2008;15(4):357-363. doi:10.1016/j.ultsonch.2007.09.008

35. Suryawanshi PG, Bhandari VM, Sorokhaibam LG, Ruparelia JP, Ranade V V. Solvent degradation studies using hydrodynamic cavitation. Environ Prog Sustain Energy. 2018;37(1):295-304. doi:10.1002/ep.12674

36. Sarvothaman VP, Nagarajan S, Ranade V V. Treatment of Solvent-Contaminated Water Using VortexBased Cavitation : In fl uence of Operating Pressure Drop, Temperature, Aeration, and Reactor Scale. Ind Eng Chem Res. 2018;57:9292-9304. doi:10.1021/acs.iecr.8b01688

37. Jain P, Bhandari VM, Balapure K, Jena J, Ranade V V., Killedar DJ. Hydrodynamic cavitation using vortex diode: An efficient approach for elimination of pathogenic bacteria from water. $J$ Environ Manage. 2019;242(April):210-219. doi:10.1016/j.jenvman.2019.04.057

38. Loraine G, Chahine G, Hsiao CT, Choi JK, Aley P. Disinfection of gram-negative and gram-positive bacteria using DynaJets ${ }^{\circledR}$ hydrodynamic cavitating jets. Ultrason Sonochem. 2012;19(3):710-717. doi:10.1016/j.ultsonch.2011.10.011

39. Delaney P. Oxidative Desulphurisation Using Vortex Based Hydrodynamic Cavitation with Water Dispersed in Organic Phase. In: 18 AlChE Annual Meeting, Pittsburgh. ; 2018.

40. Šarc A, Stepišnik-Perdih T, Petkovšek M, Dular $M$. The issue of cavitation number value in studies of water treatment by hydrodynamic cavitation. Ultrason Sonochem. 2017;34:51-59. doi:10.1016/j.ultsonch.2016.05.020

41. Simpson A, Ranade VV. Modelling of hydrodynamic cavitation with orifice: Influence of different orifice designs. Chem Eng Res Des. 2018;136. doi:10.1016/j.cherd.2018.06.014

42. Simpson A, Ranade V V. Modeling Hydrodynamic Cavitation in Venturi: Influence of Venturi Configuration on Inception and Extent of Cavitation. AIChE J. 2019;65(1):421-433. doi:10.1002/aic.16411

43. Kozák J, Rudolf $P$, Hudec M, Štefan D, Forman M. Numerical and Experimental Investigation of the Cavitating Flow Within Venturi Tube. J Fluids Eng. 2018;141(4):041101. doi:10.1115/1.4041729

44. $\quad$ Ranade et al. US Patent No. US 9,422,952 B2. 2016.

45. Simpson A, Ranade V V. Modelling of Hydrodynamic Cavitation with Orifice: Influence of different orifice designs. Chem Eng Res Des. 2018. doi:10.1016/j.cherd.2018.06.014

46. Pandare A, Ranade V V. Flow in vortex diodes. Chem Eng Res Des. 2015;102:274-285.

47. Simpson AT, Ranade VV. Flow Characteristics of Vortex Based Cavitaion Devices. AlChE J. 2019. doi:10.1002/aic.16675

48. Schindelin J, Arganda-Carreras I, Frise E, et al. Fiji: an open-source platform for biological-image analysis. Nat Methods. 2012;9(7):676-682. doi:10.1038/nmeth.2019

49. Sauer J, Schnerr GH. Unsteady cavitating flow: a new cavitation model based on a modified front capturing method and bubble dynamics. In: FEDSM'00, ASME Fluids Engineering Summer Conference. American Society of Mechanical Engineers, New York, N.Y.; 2000:11-15.

50. Zwart PJ, Gerber AG, Belamri T. A Two-Phase Flow Model for Predicting Cavitation Dynamics. In: Fiifth International Conference on Multiphase Flow, Yokohama, Japan. ; 2004.

51. Singhal AK, Athavale MM, Li H, Jiang Y. Mathematical Basis and Validation of the Full Cavitation Model. J Fluids Eng. 2002;124(3):617. doi:10.1115/1.1486223

52. Sato K, Taguchi Y, Hayashi S. High Speed Observation of Periodic Cavity Behavior in a ConvergentDivergent Nozzle for Cavitating Water Jet. 2013;2013(October):102-107.

53. Reboud J-L, Stutz B, Coutier O. Two-phase flow structure of cavitation: Experiment and modelling of unsteady effects. In: Thirds International Symposium on Cavitation. Vol 2017. Université Joseph Fourier; 1998:203-208.

54. Charrière B, Goncalves E. Numerical investigation of periodic cavitation shedding in a Venturi. Int J Heat 
Fluid Flow. 2017;64:41-54. doi:10.1016/j.ijheatfluidflow.2017.01.011

55. Charriere B, Decaix J, Goncalves E. A comparative study of cavitation models in a Venturi flow. Eur J Mech B/Fluids. 2015;49(PA):287-297. doi:10.1016/j.euromechflu.2014.10.003

56. Li D-Q, Grekula M, Lindell P. A modified SST k-lomega turbulence model to predict the steady and unsteady sheet cavitation on 2D and 3D hydrofoils. In: 7th International Symposium on Cavitation. ; 2009:1-13.

57. Hreiz R, Gentric C, Midoux N. Numerical investigation of swirling flow in cylindrical cyclones. Chem Eng Res Des. 2011;89(12):2521-2539. doi:10.1016/j.cherd.2011.05.001

58. Yin J, Li J, Ma Y, Li H, Liu W, Wang D. Study on the Air Core Formation of a Gas-Liquid Separator. J Fluids Eng. 2015;137(9):091301. doi:10.1115/1.4030198

59. Arndt REA, Arakeri VH, Higuchi H. Some observations of tip-vortex cavitation. J Fluid Mech. 1991;229:269-289. doi:10.1017/S0022112091003026

60. Arndt R, Pennings P, Bosschers J, van Terwisga T. The singing vortex. Interface Focus. 2015;5(5):1-11. doi:10.1098/rsfs.2015.0025

61. Sarvothaman VP, Simpson AT, Ranade VV. Modelling of vortex based hydrodynamic cavitation reactors. Chem Eng J. 2018;(xxxx):0-1. doi:10.1016/j.cej.2018.08.025

62. Menter FR, Kuntz M, Langtry R. Ten Years of Industrial Experience with the SST Turbulence Model.pdf. Turbul Heat Mass Transf. 2003;4(1):625-632.

63. Kato $M$, Launder $B$. The modeling of turbulent flow around stationary and vibrating square cylinders. In: Ninth Symposium on Turbulent Shear Flows. Springer Berlin Heidelberg; 1993:10.4.1-10.4.6. doi:10.1007/s13398-014-0173-7.2

64. Zaman E, Vakil A, Martinez M, Olson J. An Integral Criterion for Turbulence Suppression in Swirling Flows. Canad. 2018. doi:10.1002/cjce.23145

65. Chen L, Zhang L, Peng X, Shao X. Influence of water quality on the tip vortex cavitation inception. Phys Fluids. 2019;31(2):023303. doi:10.1063/1.5053930 
TOC Graphics

Without Swirl

Cavitation at wall
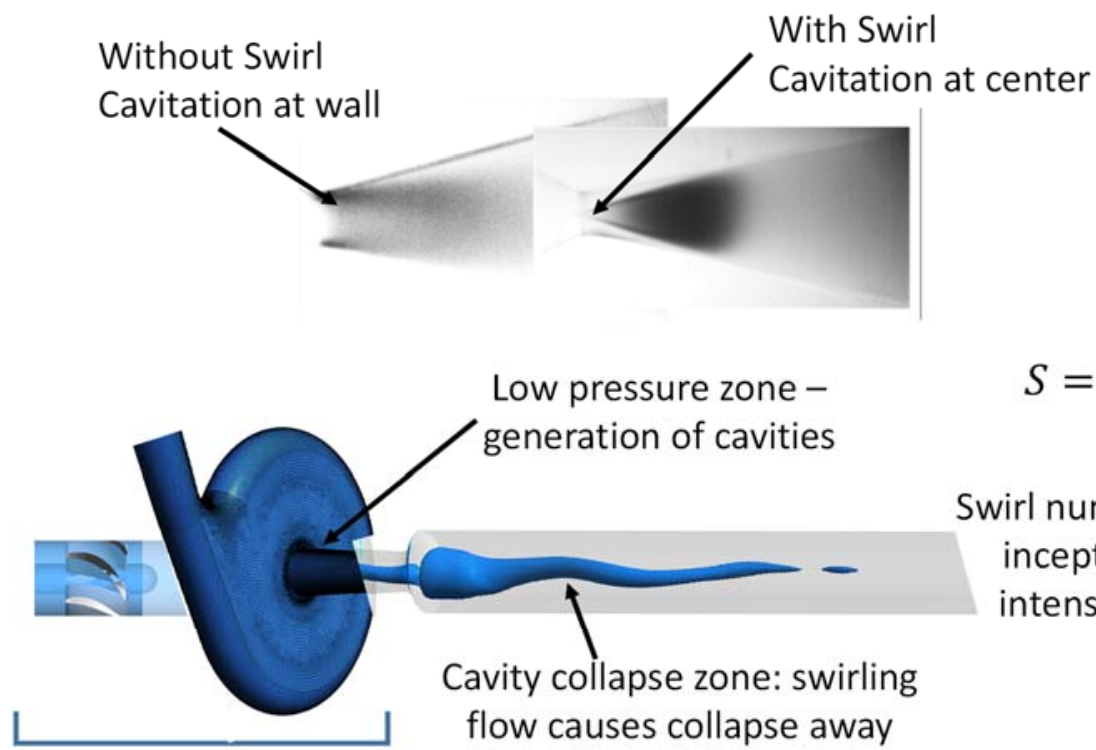

Low pressure zone generation of cavities

$$
S=\frac{u_{\theta \max }}{\overline{u_{t}}}
$$

Swirl number influences inception, extent and intensity of cavitation

Swirler before Inlet flow causes collapse away

or Tangential Inlet

CFD models provide useful insights to compare different hydrodynamic cavitation devices 